\title{
Existence, uniqueness and regularity for nonlinear parabolic equations with nonlocal terms
}

\author{
Nathaël ALIBAUD \\ Université Montpellier II \\ Département de mathématiques \\ CC 051, Place E. Bataillon \\ 34095 Montpellier cedex 5, France \\ e-mail: alibaud@math.univ-montp2.fr
}

\begin{abstract}
We study evolution equations that are fully nonlinear, degenerate parabolic, nonlocal and nonmonotone. The major difficulty lies in nonmonotonicity, i.e. in the fact that no comparison principle can be obtained. This implies that the classical method used to prove existence in the context of fully nonlinear degenerate equations, namely Perron's one, does not apply. We thus need to use a fixed point argument and to get suitable $a$ priori estimates, we need a refined version of classical continuous dependance estimates. This technical result is of independent interest. We also obtain results such as uniform or Hölder continuity of the solution.

2000 Mathematics Subject Classification: 35A05, 35B30, 35B65, 35G20, 35K65, 47G20

Key words: nonlinear degenerate parabolic integro-partial differential equation; nonmonotone equation; viscosity solution; continuous dependance estimate; Hölder estimate.
\end{abstract}

\section{Introduction}

In recent years there has been an interest in developing viscosity solutions theory for parabolic integro-PDEs. Particularly equations that occur in the theory of optimal control of jump-diffusion (Lévy) processes $[7,14,16,2,11,13]$. The use of viscosity solutions is appropriately chosen because most of these equations are degenerate or fully nonlinear. In general, existence is proved by Perron's method with the help of a comparison principle. But in few other many applications, like signal [4], there is no such a comparison principle. The equations are then said to 
be nonmonotone. The notion of viscosity solution can still be used but existence must be proved by classical fixed point methods. Our purpose is to find a general framework to treat these difficulties that are degeneracy, nonlinearity, presence of nonlocal terms and nonmonotonicity. For other recent works on nonmonotone equations, we refer the reader to $[1,3,12]$ which study equations that are involved in the theory of dislocation.

Let us present our mathematical framework. We are interested in existence, uniqueness and regularity of viscosity solution of fully nonlinear degenerate parabolic integro-PDEs of the form

$$
\begin{aligned}
\partial_{t} u+F\left(t, x, u, D u, D^{2} u, g[u]\right) & =0 \text { in } Q_{T}, \\
u(0, .) & =u_{0} \text { on } \mathbb{R}^{N},
\end{aligned}
$$

where $\left.Q_{T}:=\right] 0, T\left[\times \mathbb{R}^{N}, F:[0, T] \times \mathbb{R}^{N} \times \mathbb{R} \times \mathbb{R}^{N} \times \mathbb{S}^{N} \times \mathbb{R}^{M} \rightarrow \mathbb{R}\right.$ is a given functional nonincreasing with respect to (w.r.t. for short) the $D^{2} u$-variable and $g[u]$ is a nonlocal term. Here $\mathbb{S}^{N}$ denotes the space of symmetric $N \times N$ real valued matrices. We investigate the case where no monotonicity assumption w.r.t. the nonlocality is assumed (see Remark 3.1 for more details); this led us to consider a class of nonlocal term of order 0 satisfying a Lipschitz condition of the form

$$
\sup _{[0, t] \times \mathbb{R}^{N}}|g[u]-g[v]|(\tau, x) \leq C \sup _{[0, t] \times \mathbb{R}^{N}}|u-v|(\tau, x),
$$

where $C$ does not depend on $t, u$ and $v$. Examples of nonlocal terms satisfying (1.3) are nonlinear integral operators of the form

$$
\int_{\mathbb{R}^{N}} M(t, x, z, u(t, x), u(t, x+z)) d \mu_{t, x}(z),
$$

where $\sup _{Q_{T}}\left|\mu_{t, x}\right|\left(\mathbb{R}^{N}\right)<+\infty$ and $M$ is Lipschitz w.r.t. its two last arguments. Further examples are given by integral operators in both time and space variables such as

$$
g[u](t)=S(t) v_{0}+\int_{0}^{t} S(t-\tau) f(u(\tau)) d \tau,
$$

where $S($.$) is a semigroup generated by a linear operator A, f: \mathbb{R} \rightarrow \mathbb{R}$ is Lipschitz and $v_{0}$ is a given initial condition. Then, the Cauchy problem (1.1)-(1.2) is equivalent to the following system:

$$
\begin{aligned}
\partial_{t} u+F\left(t, x, u, D u, D^{2} u, v\right) & =0 \text { in } Q_{T}, \\
u(0, .) & =u_{0} \text { on } \mathbb{R}^{N}, \\
\frac{d}{d t} v+A v & =f(u) \text { in }] 0, T[, \\
v(0) & =v_{0} .
\end{aligned}
$$


When $A$ is the Laplacian operator, for example, the associated semigroup $S($.$) is$ defined by

$$
S(t) v:=G(t) * v,
$$

where $*$ designs the convolution product in $\mathbb{R}^{N}$ and $G(t)(x)$ is the Green Kernel.

As far as the Hamiltonian $F$ is concerned, we first study the case where it is Lipschitz w.r.t. the $g[u]$-variable and next the case where there is a coupling between $g[u]$ and the derivatives of $u$. The last one case can be seen as a generalization of $[4]$ which treats the nonmonotone equation

$$
\partial_{t} u-f(D G * u(t, .)) \operatorname{tr}\left(A(D u) D^{2} u\right)=0 \text { in } Q_{T},
$$

for $f>0$ Lipschitz-continuous, $G$ which is a Gaussian function and $A \geq 0$ bounded continuous on $\mathbb{R}^{N}-\{0\}$. In fact, our technics combined with these ones used to treat the mean curvature flow by the level set method could allow to treat discontinuous Hamiltonians at $D u=0$ and such that

$$
F^{*}(t, x, r, 0,0, \lambda)=F_{*}(t, x, r, 0,0, \lambda) .
$$

But, for the sake of clarity we have chosen to present only the continuous case. An illustrating example of what kind of coupling can be considered is the following quasilinear Hamiltonian

$$
F(t, x, r, p, X, \lambda)=H(t, x, r, p, \lambda)-\operatorname{tr}\left({ }^{t} \sigma(t, x, p, \lambda) \sigma(t, x, p, \lambda) X\right),
$$

where $H$ and $\sigma$ are Lipschitz w.r.t. $(x, \lambda)$ respectively locally and globally in $p$.

Another interest of this paper are so-called continuous dependence estimates (see Theorem 4.1) for local parabolic equations which allow to obtain lots of needed a priori estimates. This can be seen as a generalization of results of Souganidis [15, Proposition 1.4] for first-order equations and of Jakobsen and Karlsen [10, Theorem 3.1] for second-order equations. Let us recall that some of their applications are a priori Lipschitz and Hölder estimates. In our setting, their results are not sufficient, in particular they do not permit to prove that the function is uniformly continuous. This improved version seems to us of independent interest.

The rest of this paper is organized as follows: in Section 2, we introduce the definitions and notations that will be used throughout this paper. In Section 3 , we state our results and prove them in Section 4. The last one section also contains our continuous dependance estimate for local equations.

\section{Preliminaries}

Throughout the paper, we will use the notations that follow. For $a, b \in \mathbb{R}$, we let $a \vee b$ denote the real $\max \{a, b\}$. We let $a^{+}$denote the real $a \vee 0$. Let $k$ be an integer. For $x \in \mathbb{R}^{k}$, we let $|x|$ denote the Euclidean norm of $x$. We let $Q_{T}$ denote the cylinder $] 0, T\left[\times \mathbb{R}^{N}\right.$. We let $\mathbb{M}^{N}$ denote the space of $N \times N$ real valued matrices 
and $\mathbb{S}^{N}$ the space of such matrices which are symmetric. For every $X, Y \in \mathbb{S}^{N}$, we say that $X \leq Y$ when $\langle X \xi, \xi\rangle \leq\langle Y \xi, \xi\rangle$ for all $\xi \in \mathbb{R}^{N}$. The notation $\langle.,$.$\rangle is$ the Euclidean scalar product of $\mathbb{R}^{N}$. Let $\left(E, d_{E}\right)$ be a metric space. The closed ball of $E$ centered at $x$ and of radius $R$ is denoted by $\bar{B}_{E}(x, R)$.

Let us now introduce some functional vector spaces. Consider $\mu \in] 0,1]$ and $u: \bar{Q}_{T} \rightarrow \mathbb{R}^{k}$. Define

$$
\begin{aligned}
\|u\|_{\infty} & :=\sup _{t \in[0, T], x \in \mathbb{R}^{N}}|u(t, x)| \\
{[u]_{\mu} } & :=\sup _{t \in[0, T], x, y \in \mathbb{R}^{N}, x \neq y} \frac{|u(t, x)-u(t, y)|}{|x-y|^{\mu}}, \\
\|u\|_{\mu} & :=\|u\|_{\infty}+[u]_{\mu} .
\end{aligned}
$$

We let $C_{b}\left(\bar{Q}_{T}, \mathbb{R}^{k}\right)$ and $C_{b}^{0, \mu}\left(\bar{Q}_{T}, \mathbb{R}^{k}\right)$ denote the spaces of continuous functions $u: \bar{Q}_{T} \rightarrow \mathbb{R}^{k}$ such that $\|u\|_{\infty}<+\infty$ and $\|u\|_{\mu}<+\infty$, respectively. We let $B U C\left(\bar{Q}_{T}, R^{k}\right)$ denote the space of bounded uniformly continuous functions $u$. When $k=1$, we let $C_{b}\left(\bar{Q}_{T}\right), C_{b}^{0, \mu}\left(\bar{Q}_{T}\right)$ and $B U C\left(\bar{Q}_{T}\right)$ denote the preceding spaces. Consider a function $h: \mathcal{O} \subseteq \mathbb{R}^{k} \rightarrow \mathbb{R}$ and a nonnegative real $\alpha$. We let $\omega_{\alpha}(h)$ denote the modulus of continuity of size $\alpha$ of $h$. That is to say,

$$
\omega_{\alpha}(h)=\sup _{x, y \in \mathcal{O},|x-y| \leq \alpha}|h(x)-h(y)| .
$$

We call modulus a function $m: \mathbb{R}^{+} \rightarrow \mathbb{R}^{+}$such that $m$ is continuous, nondecreasing, $m(0)=0$ and such that $m\left(\alpha_{1}+\alpha_{2}\right) \leq m\left(\alpha_{1}\right)+m\left(\alpha_{2}\right)$ for all nonnegative reals $\alpha_{1}$ and $\alpha_{2}$.

Following [9], we now recall the notion of viscosity solution. This last one notion can be defined for discontinuous locally bounded functions and Hamiltonians. Here, we only need the continuous case. Consider the following general equation:

$$
\partial_{t} u+G\left(t, x, u, D u, D^{2} u\right)=0 \text { in } Q_{T},
$$

where the Hamiltonian $G:[0, T] \times \mathbb{R}^{N} \times \mathbb{R} \times \mathbb{R}^{N} \times \mathbb{S}^{N} \rightarrow \mathbb{R}$ is continuous and nonincreasing w.r.t. its last argument. For each bounded continuous function $u$ : $\bar{Q}_{T} \rightarrow \mathbb{R}$ and each subset $\mathcal{O}$ of $\bar{Q}_{T}$, we let $\mathcal{P}_{\mathcal{O}}^{2,+(-)} u(t, x)$ denote the second-order parabolic superjet (subjet) of $u$ at $(t, x) \in \mathcal{O}$ relatively to $\mathcal{O}$. Let us recall that $(a, p, X) \in \mathcal{P}_{\mathcal{O}}^{2,+(-)} u(t, x)$ if and only if (iff for short)

$$
\begin{aligned}
u(s, y) \leq & (\geq) u(t, x)+a s+\langle p, y-x\rangle \\
& +\left\langle\frac{1}{2} X(y-x), y-x\right\rangle+o\left(|s-t|+|y-x|^{2}\right)
\end{aligned}
$$

as $\mathcal{O} \ni(s, y) \rightarrow(t, x)$. We let simply $\mathcal{P}^{2,+(-)} u(t, x)$ denote the semijet $\mathcal{P}_{Q_{T}}^{2,+(-)} u(t, x)$. 
Definition 2.1 Let $u$ belong to $C\left(\bar{Q}_{T}\right)$.

i) The function $u$ is a viscosity subsolution of (2.1) iff for every $(t, x) \in Q_{T}$ and $(a, p, X) \in \mathcal{P}^{2,+} u(t, x)$,

$$
a+G(t, x, u, p, X) \leq 0
$$

ii) The function $u$ viscosity supersolution of (2.1) iff for every $(t, x) \in Q_{T}$ and $(a, p, X) \in \mathcal{P}^{2,-} u(t, x)$,

$$
a+G(t, x, u, p, X) \geq 0
$$

iii) The function $u$ viscosity solution of (2.1) iff it is both a viscosity sub- and supersolution of (2.1).

Remark 2.1 Define the closure $\overline{\mathcal{P}}_{\mathcal{O}}^{2,+\left({ }^{-}\right)} u(t, x)$ as the set of $(a, p, X) \in \mathbb{R} \times \mathbb{R}^{N} \times$ $\mathbb{S}^{N}$ such that, there are $\left(t_{n}, x_{n}\right) \in \mathcal{O}$ and $\left(a_{n}, p_{n}, X_{n}\right) \in \mathcal{P}_{\mathcal{O}}^{2,+(-)} u\left(t_{n}, x_{n}\right)$ such that $u\left(t_{n}, x_{n}\right) \rightarrow u(t, x)$ and $\left(t_{n}, x_{n}, a_{n}, p_{n}, X_{n}\right) \rightarrow(t, x, a, p, X)$. In fact, penalization technics used in [9] allow to prove that the definitions above are still true by replacing $Q_{T}$ by $\left.\left.\mathcal{O}:=\right] 0, T\right] \times \mathbb{R}^{N}$ and $\mathcal{P}^{2,(+)}{ }^{-} u(t, x)$ by $\overline{\mathcal{P}}_{\mathcal{O}}^{2,(+)-} u(t, x)$.

In a similar way, we can define the notion of continuous viscosity semisolution of the following differential equation:

$$
\dot{f}+G(f)=0 \text { in }] 0, T[
$$

where $f:[0, T] \rightarrow \mathbb{R}$ is the unknown function, and $G \in C([0, T])$ is given. Then, the notion of parabolic semijet is replaced by the notion of first-order Fréchet semidifferential. Consider $t \in] 0, T$. Let us recall that $a \in \partial^{1,+(-)} f(t)$ iff

$$
f(s) \leq(\geq) f(t)+a s+o(|s-t|),
$$

as $s \rightarrow t$.

Let us now define the notion of viscosity solution of (1.1) which is used in our paper. Consider a continuous Hamiltonian $F$ and a nonlocal term $g[]:. C_{b}\left(\bar{Q}_{T}\right) \rightarrow$ $C\left(\bar{Q}_{T}, \mathbb{R}^{M}\right)$. Let $F^{(u)}:[0, T] \times \mathbb{R}^{N} \times \mathbb{R} \times \mathbb{R}^{N} \times \mathbb{S}^{N} \rightarrow \mathbb{R}$ denote the functional defined by

$$
F^{(u)}(t, x, r, p, X)=F(t, x, r, p, X, g[u]) .
$$

Note that $F^{(u)}$ is continuous when $u$ is bounded continuous. Consider the following equation in $w$ :

$$
\partial_{t} w+F^{(u)}\left(t, x, w, D w, D^{2} w\right)=0 \text { in } Q_{T} .
$$

Definition 2.2 A function $u \in C_{b}\left(\bar{Q}_{T}\right)$ is a viscosity solution of (1.1) iff it is a viscosity solution of $(2.2)$. 


\section{Main results}

Let us state our main results. We first consider nonlocal term uncoupled with the derivatives of $u$ and next we study the case where there is a coupling. All the constants appearing in this section are noted $C^{F}$ (resp. $C^{g}$ ) when depending on the Hamiltonian $F$ (resp. the nonlocal term $g[$.$] ) and C_{R}^{F}$ when also depending on a real number $R$.

\subsection{Uncoupled nonlocal term}

Let us consider a class of nonlocal terms $g[$.] that satisfy the following conditions:

(H1) The operator $g[$.$] is well-defined from C_{b}\left(\bar{Q}_{T}\right)$ into $C_{b}\left(\bar{Q}_{T}, \mathbb{R}^{M}\right)$ (in particular, $\left.\|g[0]\|_{\infty} \leq C^{g}<+\infty\right)$.

(H2) If $u$ is uniformly continuous w.r.t. $x$ independently of $t$, then so is $g[u]$.

(H3) There exists a constant $C^{g} \geq 0$ such that for every $u, v \in C_{b}\left(\bar{Q}_{T}\right)$ and $t \in[0, T]$,

$$
\sup _{\tau \in[0, t]}\|g[u](\tau, .)-g[v](\tau, .)\|_{\infty} \leq C^{g} \sup _{\tau \in[0, t]}\|u(\tau, .)-v(\tau, .)\|_{\infty} .
$$

As far as $F$ is concerned, let us assume the following conditions:

(H4) The Hamiltonian $F$ is continuous and for each $R \geq 0, F$ is uniformly continuous w.r.t. the $\left(D u, D^{2} u\right)$-variable, independently of the others, on $[0, T] \times \mathbb{R}^{N} \times[-R, R] \times \bar{B}_{\mathbb{R}^{N}}(0, R) \times \bar{B}_{\mathbb{S}^{N}}(0, R) \times \bar{B}_{\mathbb{R}^{M}}(0, R)$.

(H5) The Hamiltonian $F$ is nondecreasing w.r.t. the $u$-variable.

There is a modulus $m_{R}($.$) , depending on a real number R$, such that for every $\varepsilon>0, t \in[0, T], x, y \in \mathbb{R}^{N}, r \in[-R, R], X, Y \in \mathbb{S}^{N}$ and $\lambda \in \bar{B}_{\mathbb{R}^{M}}(0, R)$,

(H6) if

$$
-\frac{3}{\varepsilon} \leq\left(\begin{array}{cc}
X & 0 \\
0 & -Y
\end{array}\right) \leq \frac{3}{\varepsilon}\left(\begin{array}{cc}
I & -I \\
-I & I
\end{array}\right)
$$

then

$$
F\left(t, y, r, \frac{x-y}{\varepsilon}, Y, \lambda\right)-F\left(t, x, r, \frac{x-y}{\varepsilon}, X, \lambda\right) \leq m_{R}\left(|x-y|+\frac{|x-y|^{2}}{\varepsilon}\right) .
$$

(H7) The Hamiltonian $F$ is Lipschitz w.r.t. the $g[u]$-variable, independently of the others, with a Lipschitz constant that is denoted by $C^{F}$.

(H8) $\sup _{\bar{Q}_{T}}|F(t, x, 0,0,0,0)| \leq C^{F}<+\infty$. 
Remark 3.1 Assumptions (H4)-(H6) and (H8) are classical when studying local equations (see [9]). Monotonicity assumptions w.r.t. the nonlocality (see $[14,16$, $2,11]$ and remarks in Example 3.1 below) are replaced here by (H3) and (H7). Assumption (H2) is necessary to solve (1.1)-(1.2) after having frozen the nonlocal part. If we strengthen (H4), then we can actually omit (H2) to solve (1.1)-(1.2) in the space of bounded continuous functions (to see this, one could combine technics used in [2] with our technics).

Then we have the following result.

Theorem 3.1 (Existence, uniqueness and BUC-regularity) Assume (H1)-(H8). Let $u_{0}$ belong to $B U C\left(\mathbb{R}^{N}\right)$. Then there exists a unique $u \in B U C\left(\bar{Q}_{T}\right)$ such that $u$ is a viscosity solution of (1.1)-(1.2). Moreover, for each $t \in[0, T]$

$$
\|u(t, .)\|_{\infty} \leq\left(\left\|u_{0}\right\|_{\infty}+C t\right) e^{\gamma_{0} t}
$$

where $C=C^{F}+C^{F} C^{g}$ and $\gamma_{0}=C^{F} C^{g}$.

Remark 3.2 This theorem is also a regularity result, since we get the uniform continuity of the solution. Moreover, the assumptions (in particular (H2) and (H6)) seem to us quite general to get this.

Example 3.1 Suitable assumptions under which nonlocal terms of the form (1.4) or (1.5) satisfy (H1)-(H3) can easily be determinate by the readers. Some simple illustrating examples of such nonlocal terms are:

- convolution operators $K * u(t,$.$) , where K \in L^{1}\left(\mathbb{R}^{N}\right)$;

- Lévy operators of the form $-\int_{\mathbb{R}^{N}} u(t, x+z)-u(t, x) d \mu(z)$, where $\mu$ is a bounded Borel measure (under suitable assumptions to ensure (H2), $\mu$ can also depend on $(t, x))$;

- Volterra operators of the form $-\int_{0}^{t} B(t-s) u(s, x) d s$, where $B \in L^{1}(0, T)$.

Note that if the measure and the kernels above are nonnegative and if $F$ is nondecreasing w.r.t. the $g[u]$-variable then $(1.1)$ is monotone (see $[16,2,11])$; but, in the general case (1.1) can become nonmonotone.

We are now interested in Hölder regularity of $u$ w.r.t. $x$. For $\mu \in] 0,1]$, let us consider the following condition:

(H2)' There exists a constant $C^{g} \geq 0$ such that for every $u \in C_{b}^{0, \mu}\left(\bar{Q}_{T}\right)$ and $t \in[0, T]$,

$$
\sup _{\tau \in[0, t]}[g[u](\tau, .)]_{\mu} \leq C^{g}\left(1+\sup _{\tau \in[0, t]}\|u(\tau, .)\|_{\mu}\right) .
$$


We let also

(H6)' denote Assumption (H6) for $m_{R}\left(|x-y|+\frac{|x-y|^{2}}{\varepsilon}\right)=C_{R}^{F}\left(|x-y|^{\mu}\right.$ $\left.+\frac{|x-y|^{2}}{\varepsilon}\right)$

where $C_{R}^{F}$ is a nonnegative constant that depends on $R$. Let us state our regularity result.

Theorem 3.2 (Hölder regularity) Assume that (H1), (H2)', (H3)-(H5), (H6)', (H7) and (H8) hold true for any $\mu \in] 0,1]$. Let $u$ be the unique viscosity solution of (1.1)-(1.2). Define $R=\|u\|_{\infty}$ and $R^{g}=C^{g}(1+R)$. Then for each $t \in[0, T]$,

$$
[u(t, .)]_{\mu} \leq\left(4\left[u_{0}\right]_{\mu} e^{\frac{\mu}{2} \bar{\gamma} t}+C_{R}\right) e^{\gamma_{1}(t) t}
$$

where $\bar{\gamma}=2\left(C_{R \vee R^{g}}^{F}+1\right), \gamma_{1}(t)=\left(4 C^{F} C^{g} e^{\frac{\mu}{2} \bar{\gamma} t}\right)^{\frac{2}{2-\mu}}$ and $C_{R}=\left(C^{F} C^{g}\right)^{-1}\left(C_{R \vee R^{g}}^{F}+\right.$ $\left.C^{F} R^{g}\right)$.

\subsection{Nonlocal term coupled with the derivatives}

Now, we strengthen (H2)' by:

(H2)" There exists a constant $C^{g} \geq 0$ such that for every $u \in C_{b}\left(\bar{Q}_{T}\right)$ and $t \in[0, T]$,

$$
\sup _{\tau \in[0, t]}[g[u](\tau, .)]_{1} \leq C^{g}\left(1+\sup _{\tau \in[0, t]}\|u(\tau, .)\|_{\infty}\right) .
$$

Assumptions (H6)' and (H7) on $F$ are relaxed by:

(H9) There exists a constant $C^{F} \geq 0$, and for each $R \geq 0$ there exists a constant $C_{R}^{F} \geq 0$ such that, for every $\varepsilon>0, t \in[0, T], x, y \in \mathbb{R}^{N},|r| \leq R, X, Y \in \mathbb{S}^{N}$ and $|\lambda|,|\mu| \leq R$, if (3.1) holds true then

$$
\begin{aligned}
& F\left(t, y, r, \frac{x-y}{\varepsilon}, Y, \lambda\right)-F\left(t, x, r, \frac{x-y}{\varepsilon}, X, \mu\right) \leq \\
& C_{R}^{F}\left(|x-y|+\frac{|x-y|^{2}}{\varepsilon}\right)+C^{F}\left(|\lambda-\mu|+|\lambda-\mu| \frac{|x-y|}{\varepsilon}+\frac{|\lambda-\mu|^{2}}{\varepsilon}\right) .
\end{aligned}
$$

Remark 3.3 Let us comment assumption (H9), Under (H6)' (with $\mu=1$ ) and (H7), we have

$$
\begin{gathered}
F\left(t, y, r, \frac{x-y}{\varepsilon}, Y, \lambda\right)-F\left(t, x, r, \frac{x-y}{\varepsilon}, X, \mu\right) \\
\leq C_{R}^{F}\left(|x-y|+\frac{|x-y|^{2}}{\varepsilon}\right)+C^{F}|\lambda-\mu| .
\end{gathered}
$$


The role of the new term $C^{F}|\lambda-\mu| \frac{|x-y|}{\varepsilon}+C^{F} \frac{|\lambda-\mu|^{2}}{\varepsilon}$ that appears in (H9) can be illustrate by the following simple example of Hamiltonian:

$$
F(t, x, r, p, X, \lambda)=\lambda|p|-\operatorname{tr}(A(\lambda) X),
$$

where $A={ }^{t} \sigma \sigma$ and $\sigma$ is Lipschitz.

Let us state our last result.

Theorem 3.3 (Existence, uniqueness and Lipschitz regularity) Let us assume (H1), (H2)", (H3)-(H5), (H8) and (H9). Then for each $u_{0} \in W^{1, \infty}\left(\mathbb{R}^{N}\right)$ there exists a unique $u \in C_{b}\left(\bar{Q}_{T}\right)$ such that $u$ is a viscosity solution of (1.1) and (1.2). Moreover, defining $R=\|u\|_{\infty}$ and $R^{g}=C^{g}(1+R)$, Estimate (3.2) still holds true and for each $t \in[0, T]$

$$
[u(t, .)]_{1} \leq\left(2\left[u_{0}\right]_{1}+C_{R} t\right) e^{\frac{\bar{\gamma}}{2} t}
$$

where $\bar{\gamma}=2\left(C_{R \vee R^{g}}^{F}+C^{F} C^{g}\left(1+C^{g}+R+C^{g} R^{2}\right)\right)+1$ and $C_{R}=\left(C_{R \vee R^{g}}^{F}+C^{F} R^{g}\right)$.

Example 3.2 Simple assumptions under which quasilinear Hamiltonians of the form (1.6) satisfy (H9) are the following: there are nonnegative constants $C_{i}$ $(i=1,2)$ and for each $R \geq 0$ there exists $C_{R} \geq 0$ such that:

For every $t \in[0, T], x \in \mathbb{R}^{N}, r \in \mathbb{R}, p \in \mathbb{R}^{N}, \lambda \in \mathbb{R}^{M}$,

$$
\left|\partial_{\lambda} H(t, x, r, p, \lambda)\right| \leq C_{1}(1+|p|) \text { and }\left|\partial_{\lambda} \sigma(t, x, p, \lambda)\right| \leq C_{2} .
$$

If $|r|,|\lambda| \leq R$, then $\left|\partial_{x} H(t, x, r, p, \lambda)\right| \leq C_{R}(1+|p|)$ and $\left|\partial_{x} \sigma(t, x, p, \lambda)\right| \leq C_{R}$.

\section{Proofs of the results}

In this section, we prove the preceding results. This section is organized as follows: in Subsection 4.1, we state a technical result (Theorem 4.1). This result is proved in Subsection 4.4. Subsection 4.2 is devoted to the proofs of Theorems 3.1 and 3.2. Theorem 3.3 is proved in Subsection 4.3.

\subsection{Continuous dependence estimates for local parabolic equations}

To state our technical result, we have to introduce some notations. Let us consider equations of the form

$$
\partial_{t} u_{i}+G_{i}\left(t, x, u_{i}, D u_{i}, D^{2} u_{i}\right)=0 \text { in } Q_{T},
$$


where $G_{i}:[0, T] \times \mathbb{R}^{N} \times \mathbb{R} \times \mathbb{R}^{N} \times \mathbb{S}^{N} \rightarrow \mathbb{R}$ is a given functional $(i=1,2)$. We make the following assumptions on $G_{i}$ :

The Hamiltonian $G_{i}$ is continuous and for each $R \geq 0, G_{i}$ is uniformly continuous w.r.t. the $\left(D u_{i}, D^{2} u_{i}\right)$-variables, independently of the others, on

$$
[0, T] \times \mathbb{R}^{N} \times[-R, R] \times \bar{B}_{\mathbb{R}^{N}}(0, R) \times \bar{B}_{\mathbb{S}^{N}}(0, R)
$$

There exists $\gamma \geq 0$ such that for every $t \in[0, T], x \in \mathbb{R}^{N}, r, s \in \mathbb{R}, p \in \mathbb{R}^{N}$ and $X \in \mathbb{S}^{N}$, if $r \geq s$ then

$$
G_{i}(t, x, r, p, X) \geq G_{i}(t, x, s, p, X)+\gamma(r-s) .
$$

For each $R \geq 0$, there exists a modulus $m_{R}$ (.) such that for every $\varepsilon>0, t \in[0, T]$, $x, y \in \mathbb{R}^{N}, r \in[-R, R]$ and $X, Y \in \mathbb{S}^{N}$, if (3.1) holds true then

$$
G_{i}\left(t, y, r, \frac{x-y}{\varepsilon}, Y\right)-G_{i}\left(t, x, r, \frac{x-y}{\varepsilon}, X\right) \leq m_{R}\left(|x-y|+\frac{|x-y|^{2}}{\varepsilon}\right) .
$$

Finally, we assume that for each $R \geq 0$,

$$
\sup _{(t, x, r) \in \bar{Q}_{T} \times[-R, R]}\left(G_{2}(t, x, r, 0,0)-G_{1}(t, x, r, 0,0)\right)<+\infty .
$$

Let $u_{i}$ be a bounded continuous sub- and supersolution of (4.1) for respectively $i=1$ and $i=2, \bar{\gamma}$ be a nonnegative constant (that will be appropriately chosen when using Theorem 4.1 in Subsection 4.2 and 4.3) and $\varepsilon$ be a positive real. For $t \in[0, T]$, define

$$
\begin{aligned}
m_{\varepsilon}(t):= & \sup _{(x, y) \in \mathbb{R}^{N} \times \mathbb{R}^{N}}\left(u_{1}(t, x)-u_{2}(t, y)-e^{\bar{\gamma} t} \frac{|x-y|^{2}}{2 \varepsilon}\right)^{+}, \\
\sigma_{\varepsilon}(t):= & \inf _{n \in \mathbb{N}^{*}} \sup _{d_{\varepsilon}^{n}(t)}\left(G_{2}\left(t, y, r, e^{\bar{\gamma} t} p, e^{\bar{\gamma} t} Y\right)-G_{1}\left(t, x, r, e^{\bar{\gamma} t} p, e^{\bar{\gamma} t} X\right)\right. \\
& \left.-\bar{\gamma} e^{\bar{\gamma} t} \frac{|x-y|^{2}}{2 \varepsilon}\right)^{+},
\end{aligned}
$$

where we let $d_{\varepsilon}^{n}(t)$ denote the set of $(x, y, r, p, X, Y)$ such that,

$$
\left\{\begin{array}{l}
|r| \leq\left\|u_{1}(t, .)\right\|_{\infty} \vee\left\|u_{2}(t, .)\right\|_{\infty} \\
p=\frac{x-y}{\varepsilon} \\
\text { Condition }(3.1) \text { holds true, } \\
\frac{|x-y|^{2}}{4 \varepsilon} \leq m_{2 \varepsilon}(t)-m_{\varepsilon}(t)+\frac{1}{n}
\end{array}\right.
$$

We also define

$$
\Sigma_{\varepsilon}(t):=\sup _{D_{\varepsilon}(t)}\left(G_{2}\left(\tau, y, r, e^{\bar{\gamma} \tau} p, e^{\bar{\gamma} t} Y\right)-G_{1}\left(\tau, x, r, e^{\bar{\gamma} t} p, e^{\bar{\gamma} \tau} X\right)-\bar{\gamma} e^{\bar{\gamma} \tau} \frac{|x-y|^{2}}{2 \varepsilon}\right)^{+},
$$


where we let $D_{\varepsilon}(t)$ denote the set of $(\tau, x, y, r, p, X, Y)$ such that

$$
\left\{\begin{array}{l}
r \in[0, t] \\
|r| \leq\left\|u_{1}(\tau, .)\right\|_{\infty} \vee\left\|u_{2}(\tau, .)\right\|_{\infty} \\
p=\frac{x-y}{\varepsilon} \\
\text { Condition (3.1) holds true. }
\end{array}\right.
$$

Then, we have the following result.

Theorem 4.1 Assume that $G_{i}$ satisfies (4.2)-(4.5) ( $\left.i=1,2\right)$. Assume that $u_{i} \in$ $C_{b}\left(\bar{Q}_{T}\right)$ is a sub-and a supersolution of (4.1) for $i=1$ and $i=2$, respectively. Then:

i) For each $\varepsilon>0$ the function $\sigma_{\varepsilon}($.$) is measurable and for each t \in[0, T]$,

$$
m_{\varepsilon}(t) \leq m_{\varepsilon}(0)+\int_{0}^{t} \sigma_{\varepsilon}(\tau) d \tau
$$

ii) If moreover $\gamma>0$, then for each $t \in[0, T] m_{\varepsilon}(t) \leq m_{\varepsilon}(0)+\frac{1}{\gamma} \Sigma_{\varepsilon}(t)$.

The proof of this result is given in Subsection 4.4.

\subsection{Case of an uncoupled nonlocal term}

Proof of Theorem 3.1 We use a contracting fixed point theorem. We let the reader verify that for $u \in B U C\left(\bar{Q}_{T}\right)$ the Hamiltonian $F^{(u)}$ satisfies (4.2)-(4.4). Under these assumptions, it is well-known that there is a comparison principle between semicontinuous semisolutions of (2.2). Moreover, $\sup _{\bar{Q}_{T}}\left|F^{(u)}(t, x, 0,0,0)\right|<+\infty$ and for $u_{0} \in B U C\left(\mathbb{R}^{N}\right)$ there exists a unique bounded continuous solution $w$ of (2.2) such that (1.2) is satisfied; we let $\Theta u$ denote this solution. For a proof of these results we refer the reader to [9].

First step: stability of $B U C\left(\bar{Q}_{T}\right)$ by $\Theta$. Let us prove that for all $u \in B U C\left(\bar{Q}_{T}\right)$, $\Theta u$ is uniformly continuous. We let $m_{\varepsilon}(),. \sigma_{\varepsilon}($.$) and d_{\varepsilon}^{n}($.$) denote the functions$ introduced in Subsection 4.1 for $u_{i}=u, G_{i}=F^{(u)}(i=1,2)$ and $\bar{\gamma}=0$. Let $m_{R}^{(u)}($.$) denote the modulus deriving from (4.4). For \varepsilon>0, t \in[0, T]$ and $(x, y, r, p, X, Y) \in d_{\varepsilon}^{n}(t)$

$$
F^{(u)}(t, y, r, p, Y)-F^{(u)}(t, x, r, p, X) \leq m_{R}^{(u)}\left(|x-y|+\frac{|x-y|^{2}}{\varepsilon}\right),
$$

where $R=\|\Theta u\|_{\infty}$. By the definition of $d_{\varepsilon}^{n}(t)$, we see that $\frac{|x-y|^{2}}{4 \varepsilon} \leq m_{2 \varepsilon}(t)-$ $m_{\varepsilon}(t)+\frac{1}{n}$. Thus

$$
|x-y|+\frac{|x-y|^{2}}{\varepsilon} \leq 2 \sqrt{\varepsilon} \sqrt{m_{2 \varepsilon}(t)-m_{\varepsilon}(t)+\frac{1}{n}}+4\left(m_{2 \varepsilon}(t)-m_{\varepsilon}(t)+\frac{1}{n}\right) .
$$


Let $\alpha_{\varepsilon}^{n}(t)$ denote the right hand side of this inequality and define $\alpha_{\varepsilon}(t):=\inf _{n \in \mathbb{N}^{*}}$ $\alpha_{\varepsilon}^{n}(t)$. We get

$$
F^{(u)}(t, y, r, p, Y)-F^{(u)}(t, x, r, p, X) \leq m_{R}^{(u)}\left(\alpha_{\varepsilon}^{n}(t)\right) .
$$

Taking the supremum w.r.t. $(x, y, r, p, X, Y) \in d_{\varepsilon}^{n}(t)$, we see that for all $\varepsilon>0$ and all $t \in[0, T]$,

$$
\sigma_{\varepsilon}(t) \leq \inf _{n \in \mathbb{N}^{*}} m_{R}^{(u)}\left(\alpha_{\varepsilon}^{n}(t)\right) \leq m_{R}^{(u)}\left(\alpha_{\varepsilon}(t)\right)
$$

By the item i) of Theorem 4.1, we find that for all $\varepsilon>0$ and all $t \in[0, T]$,

$$
m^{\varepsilon}(t) \leq m^{\varepsilon}(0)+\int_{0}^{T} m_{R}^{(u)}\left(\alpha_{\varepsilon}(\tau)\right) d \tau .
$$

We let $I_{\varepsilon}$ denote the integral term of this inequality. Let us recall that for each $\tau \in$ $[0, T], m_{\varepsilon}(\tau)$ is nonnegative and nondecreasing w.r.t. $\varepsilon$. The $\operatorname{limit} \lim _{\varepsilon \gtrsim_{0}} m_{\varepsilon}(\tau)$ then exists and $\lim _{\varepsilon \rightarrow 0}\left(m_{2 \varepsilon}(\tau)-m_{\varepsilon}(\tau)\right)=0$. Moreover, we know that $m_{\varepsilon}(\tau) \leq$ $2\|\Theta u\|_{\infty}$ and we infer that the family of functions $\left(m_{R}^{(u)}\left(\alpha_{\varepsilon}(.)\right)\right)_{\varepsilon>0}$ is uniformly bounded and converges to 0 pointwise as $\varepsilon \gg 0$. By Lebesgue's convergence Theorem, we deduce that $\lim _{\varepsilon \rightarrow 0} I_{\varepsilon}=0$. Since (4.6) implies that

$$
|\Theta u(t, x)-\Theta u(t, y)| \leq m_{\varepsilon}(0)+I_{\varepsilon}+\frac{|x-y|^{2}}{2 \varepsilon},
$$

for all $t \in[0, T]$, all $x, y \in \mathbb{R}^{N}$ and all $\varepsilon>0$, we have proved that $\Theta u$ is uniformly continuous w.r.t. $x$ independently of $t$.

The uniform continuity in both time and space variable is now a consequence of the following result.

Proposition 4.1 For each $\nu>0$, there exists $C_{\nu} \geq 0$ that only depends on the Hamiltonian $F,\|u\|_{\infty},\|\Theta u\|_{\infty}$ and the modulus of continuity w.r.t. the $x$-variable of $\Theta u$ and that is such that, for each $\alpha \geq 0$,

$$
\sup _{x \in \mathbb{R}^{N}} \omega_{\alpha}(\Theta u(., x)) \leq \inf _{\nu>0}\left\{\nu+C_{\nu} \alpha\right\}
$$

The proof of Proposition 4.1 is well-known for local equations (see [6, Lemma 9.1]) and can easily be adapted to nonlocal equations; for the reader's convenience, a sketch of the proof of this result is given in Appendix A. The proof of the fact that $\Theta$ maps $B U C\left(\bar{Q}_{T}\right)$ into itself is complete.

Second step: resolution of (1.1)-(1.2). Define the space

$$
E:=\left\{u \in B U C\left(\bar{Q}_{T}\right): u(0, .)=u_{0}\right\}
$$


that we endow with the distance $d_{E}(u, v)=\left\|\left(u_{1}-u_{2}\right)_{\gamma}\right\|_{\infty}$, where $\gamma:=2 C^{F} C^{g}$ and

$$
w_{\gamma}(t, x):=e^{-\gamma t} w(t, x)
$$

We know that $\Theta(E) \subseteq E$. Let us prove that $\Theta: E \rightarrow E$ is a contraction. Let $u_{i}$ belong to $E(i=1,2)$. Define $v:=\Theta u_{2}+\frac{e^{\gamma t}}{2} d_{E}\left(u_{1}, u_{2}\right)$. Let $(a, p, X)$ belong to $\mathcal{P}^{2,-} v(t, x)$. Using successively (H5), (H7) and (H3), we show that

$$
\begin{aligned}
a+F^{\left(u_{1}\right)}(t, x, v, p, X) \geq & a+F\left(t, x, \Theta u_{2}, p, X, g\left[u_{1}\right]\right), \\
\geq & a+F\left(t, x, \Theta u_{2}, p, X, g\left[u_{2}\right]\right) \\
& -C^{F}\left|g\left[u_{1}\right](t, x)-g\left[u_{2}\right](t, x)\right|, \\
\geq & a+F\left(t, x, \Theta u_{2}, p, X, g\left[u_{2}\right]\right) \\
& -C^{F} C^{g} \sup _{\tau \in[0, t]} \mid\left\|u_{1}(\tau, .)-u_{2}(\tau, .)\right\|_{\infty}, \\
\geq & a+F^{\left(u_{2}\right)}\left(t, x, \Theta u_{2}, p, X,\right)-C^{F} C^{g} e^{\gamma t} d_{E}\left(u_{1}, u_{2}\right) .
\end{aligned}
$$

Since $\left(a-C^{F} C^{g} e^{\gamma t} d_{E}\left(u_{1}, u_{2}\right), p, X\right) \in \mathcal{P}^{2,-} \Theta u_{2}(t, x)$, the viscosity inequalities applied to $\Theta u_{2}$ (supersolution of (2.2) with $u=u_{2}$ ) imply that $v$ is a supersolution of (2.2) with $u=u_{1}$. By the comparison principle, we infer that $\Theta u_{1} \leq v$ and $\left(\Theta u_{1}-\Theta u_{2}\right)_{\gamma} \leq \frac{1}{2} d_{E}\left(u_{1}, u_{2}\right)$. We can argue similarly to get the other inequality. We conclude that $\Theta$ is a contraction. Since the metric space $\left(E, d_{E}\right)$ is complete, Banach fixed point Theorem implies that the Cauchy problem (1.1)-(1.2) admits a unique viscosity solution in $B U C\left(\bar{Q}_{T}\right)$.

Third step: proof of (3.2). Estimate (3.2) will be a consequence of the following result.

Proposition 4.2 If $u$ satisfies (3.2), then $\Theta u$ also satisfies (3.2).

Proof. Let $t \in[0, T[$ and $h>0$ be such that $t+h \leq T$. A simple computation shows that the function

$$
s \rightarrow v(s):=\sup _{\tau \in[0, t]}\|\Theta u(\tau, .)\|_{\infty}+(s-t) \sup _{(\tau, x) \in \bar{Q}_{t+h}}\left|F^{(u)}(\tau, x, 0,0,0)\right|
$$

is a supersolution of $(2.2)$ in the domain $] t, t+h\left[\times \mathbb{R}^{N}\right.$. Since $\Theta u(t,.) \leq v(t)$, the comparison principle implies that

$$
\sup _{\bar{Q}_{t+h}} \Theta u \leq \sup _{\tau \in[0, t]}|| \Theta u(\tau, .) \|_{\infty}+h \sup _{(\tau, x) \in \bar{Q}_{t+h}}|F(\tau, x, 0,0,0, g[u])| .
$$


Using successively (H7), (H3) and (H1) and (H8), we deduce that for all $(\tau, x) \in$ $\bar{Q}_{t+h}$,

$$
\begin{aligned}
|F(\tau, x, 0,0,0, g[u])| \leq & |F(\tau, x, 0,0,0,0)|+C^{F}|g[u](\tau, x)|, \\
\leq & |F(\tau, x, 0,0,0,0)| \\
& +C^{F}\left(\|g[0]\|_{\infty}+C^{g} \sup _{\tau^{\prime} \in[0, \tau]}\left\|u\left(\tau^{\prime}, .\right)\right\|_{\infty}\right), \\
\leq & C^{F}+C^{F} C^{g}\left(1+\sup _{\tau^{\prime} \in[0, t+h]}\left\|u\left(\tau^{\prime}, .\right)\right\|_{\infty}\right), \\
= & C+\gamma_{0} \sup _{\tau^{\prime} \in[0, t+h]}\left\|u\left(\tau^{\prime}, .\right)\right\|_{\infty},
\end{aligned}
$$

where $C$ and $\gamma_{0}$ are defined as in (3.2). We get

$$
\sup _{\bar{Q}_{t+h}} \Theta u \leq \sup _{\tau \in[0, t]}\|\Theta u(\tau, .)\|_{\infty}+h\left(C+\gamma_{0} \sup _{\tau \in[0, t+h]}\|u(\tau, .)\|_{\infty}\right) .
$$

We can argue similarly to get the other inequality. We deduce that for all $0 \leq t \leq$ $t+h \leq T$

$$
\sup _{\tau \in[0, t+h]}\|\Theta u(\tau, .)\|_{\infty} \leq \sup _{\tau \in[0, t]}\|\Theta u(\tau, .)\|_{\infty}+h\left(C+\gamma_{0} \sup _{\tau \in[0, t+h]}\|u(\tau, .)\|_{\infty}\right) .
$$

Since $\Theta u$ is uniformly continuous, the function

$$
t \in[0, T] \rightarrow f(t):=\sup _{\tau \in[0, t]}\|\Theta u(\tau, .)\|_{\infty}
$$

is continuous. Lemma 4.1 then implies that if $u$ satisfies (3.2), then $f$ is a continuous viscosity subsolution of the following equation:

$$
\left.\dot{f}=C+\gamma_{0} g \text { in }\right] 0, T[,
$$

where $g(t)$ is equal to the right hand side of (3.2). The comparison principle then completes the proof of Proposition 4.2

Let us return to the proof of (3.2). Since $\{u \in E:(3.2)$ holds true $\}$ is a nonempty closed subspace of $E$ which is stable by $\Theta$, we see that the unique fixed point of $\Theta$ belongs to this subspace. This completes the proof of (3.2) and $a$ fortiori the proof of Theorem 3.1.

Proof of Theorem 3.2 Consider the space

$$
E^{\mu}:=\{u \in E:(3.2) \text { and (3.3) hold true }\},
$$

where $R$ is any $L^{\infty}$ bound on $E^{\mu}$. 
First step: stability of $E^{\mu}$ by $\Theta$. Let us prove that $\Theta\left(E^{\mu}\right) \subseteq E^{\mu}$. Proposition 4.2 implies that for all $u \in E^{\mu}, \Theta u$ satisfies (3.2) and

$$
\|\Theta u\|_{\infty} \leq R \text {. }
$$

To prove the Hölder continuity of $\Theta u$, we have to introduce some notations. For $\gamma>0$, define

$$
\begin{aligned}
F_{\gamma}(t, x, r, p, X, \lambda) & :=e^{-\gamma t} F\left(t, x, e^{\gamma t} r, e^{\gamma t} p, e^{\gamma t} X, \lambda\right), \\
F_{\gamma}^{(u)}(t, x, r, p, X) & :=F_{\gamma}(t, x, r, p, X, g[u]) .
\end{aligned}
$$

The function $(\Theta u)_{\gamma}$, defined as in (4.8), is a viscosity solution of the following equation in $w$ :

$$
\partial_{t} w+\gamma w+F_{\gamma}^{(u)}\left(t, x, w, D w, D^{2} w\right)=0 \text { in } Q_{T} .
$$

Following [15] and [10], we derive an Hölder estimate on $(\Theta u)_{\gamma}$. Theorem 4.1 item ii), applied to

$$
G_{i}(t, x, r, p, X)=\gamma r+F_{\gamma}^{(u)}(t, x, r, p, X)
$$

and $u_{i}=(\Theta u)_{\gamma}(i=1,2)$, implies that for all $\varepsilon>0$, all $t \in[0, T]$ and all $x, y \in \mathbb{R}^{N}$,

$$
(\Theta u)_{\gamma}(t, x)-(\Theta u)_{\gamma}(t, y) \leq m_{\varepsilon}(0)+\frac{1}{\gamma} \Sigma_{\varepsilon}(t)+e^{\bar{\gamma} t} \frac{|x-y|^{2}}{2 \varepsilon} .
$$

We have

$$
\begin{aligned}
m_{\varepsilon}(0) & =\sup _{(x, y) \in \mathbb{R}^{N} \times \mathbb{R}^{N}}\left(u_{0}(x)-u_{0}(y)-\frac{|x-y|^{2}}{2 \varepsilon}\right)^{+}, \\
& \leq \sup _{(x, y) \in \mathbb{R}^{N} \times \mathbb{R}^{N}}\left(\left[u_{0}\right]_{\mu}|x-y|^{\mu}-\frac{|x-y|^{2}}{2 \varepsilon}\right)^{+}, \\
& \leq\left[u_{0}\right]_{\mu}^{\frac{2}{2-\mu}} \varepsilon^{\frac{\mu}{2-\mu}}
\end{aligned}
$$

Let us derive an upper bound on $\Sigma_{\varepsilon}(t)$. For $(\tau, x, y, r, p, X, Y) \in D_{\varepsilon}(t)$, define

$$
\begin{aligned}
I:= & F_{\gamma}^{(u)}\left(\tau, y, r, e^{\bar{\gamma} \tau} p, e^{\bar{\gamma} \tau} Y\right)-F_{\gamma}^{(u)}\left(\tau, x, r, e^{\bar{\gamma} \tau} p, e^{\bar{\gamma} \tau} X\right), \\
= & F_{\gamma}\left(\tau, y, r, e^{\bar{\gamma} \tau} \frac{x-y}{\varepsilon}, e^{\bar{\gamma} \tau} Y, g[u](\tau, y)\right) \\
& -F_{\gamma}\left(\tau, x, r, e^{\bar{\gamma} \tau} \frac{x-y}{\varepsilon}, e^{\bar{\gamma} \tau} X, g[u](\tau, x)\right) .
\end{aligned}
$$

Condition (H7) implies that

$$
\begin{aligned}
I \leq & F_{\gamma}\left(\tau, y, r, e^{\bar{\gamma} \tau} \frac{x-y}{\varepsilon}, e^{\bar{\gamma} \tau} Y, g[u](\tau, x)\right) \\
& -F_{\gamma}\left(\tau, x, r, e^{\bar{\gamma} \tau} \frac{x-y}{\varepsilon}, e^{\bar{\gamma} \tau} X, g[u](\tau, x)\right)+e^{-\gamma \tau} C^{F}|g[u](\tau, y)-g[u](\tau, x)| .
\end{aligned}
$$


By (H1) and (H3), we see that $|g[u](\tau, x)| \leq R^{g}$ where $R^{g}$ is defined as in Theorem 3.2. By the definition of $D_{\varepsilon}(t)$, we see that $e^{\gamma \tau}|r| \leq e^{\gamma \tau}||(\Theta u)_{\gamma}(\tau,.) \|_{\infty} \leq$ $\|\Theta u\|_{\infty} \leq R$ (see (4.9)). Moreover, Condition (3.1) holds true and (H6)' then implies that

$$
\begin{aligned}
I & \leq e^{-\gamma \tau} C_{R \vee R^{g}}^{F}\left(|x-y|^{\mu}+e^{(\gamma+\bar{\gamma}) \tau} \frac{|x-y|^{2}}{\varepsilon}\right)+e^{-\gamma \tau} C^{F}|g[u](\tau, y)-g[u](\tau, x)|, \\
& \leq C_{R \vee R^{g}}^{F}\left(|x-y|^{\mu}+e^{\bar{\gamma} \tau} \frac{|x-y|^{2}}{\varepsilon}\right)+C^{F} e^{-\gamma \tau}[g[u](\tau, .)]_{\mu}|x-y|^{\mu} .
\end{aligned}
$$

Using (H2)', we get

$$
I \leq C_{1}|x-y|^{\mu}+C_{2} e^{\bar{\gamma} \tau} \frac{|x-y|^{2}}{\varepsilon},
$$

where $C_{1}=C_{R \vee R^{g}}^{F}+C^{F}\left(R^{g}+C^{g} \sup _{s \in[0, t]}\left[u_{\gamma}(s, .)\right]_{\mu}\right)$ and $C_{2}=C_{R \vee R^{g}}^{F}$. Taking the supremum w.r.t. $(\tau, x, y, r, p, X, Y) \in D_{\varepsilon}(t)$, we get

$$
\Sigma_{\varepsilon}(t) \leq \sup _{\substack{\tau \in[0, t], x, y \in \mathbb{R}^{N}}}\left(C_{1}|x-y|^{\mu}+C_{2} e^{\bar{\gamma} \tau} \frac{|x-y|^{2}}{\varepsilon}-\bar{\gamma} e^{\bar{\gamma} \tau} \frac{|x-y|^{2}}{2 \varepsilon}\right)^{+} .
$$

If we take $\bar{\gamma}=2\left(C_{2}+1\right)$, then for all $t \in[0, T]$ and all $\varepsilon>0$,

$$
\Sigma_{\varepsilon}(t) \leq \sup _{r>0}\left(C_{1} r^{\mu}-\frac{r^{2}}{\varepsilon}\right)^{+} \leq C_{1}^{\frac{2}{2-\mu}} \varepsilon^{\frac{\mu}{2-\mu}} .
$$

Inequalities (4.10) and (4.11) then imply that for all $t \in[0, T]$ and all $x, y \in \mathbb{R}^{N}$,

$$
\begin{aligned}
(\Theta u)_{\gamma}(t, x)-(\Theta u)_{\gamma}(t, y) & \leq \inf _{\varepsilon>0}\left\{\left(\left[u_{0}\right]_{\mu}^{\frac{2}{2-\mu}}+\frac{C_{1}^{\frac{2}{2-\mu}}}{\gamma}\right) \varepsilon^{\frac{\mu}{2-\mu}}+e^{\bar{\gamma} t} \frac{|x-y|^{2}}{2 \varepsilon}\right\}, \\
& \leq 2\left(\left[u_{0}\right]_{\mu}^{\frac{2}{2-\mu}}+\frac{C_{1}^{\frac{2}{2-\mu}}}{\gamma}\right)^{\frac{2-\mu}{2}} e^{\frac{\mu}{2} \bar{\gamma} t}|x-y|^{\mu}, \\
& \leq 2\left(\left[u_{0}\right]_{\mu}+\frac{C_{1}}{\gamma^{\frac{2-\mu}{2}}}\right) e^{\frac{\mu}{2} \bar{\gamma} t}|x-y|^{\mu}
\end{aligned}
$$

that is to say,

$$
\begin{aligned}
& {\left[(\Theta u(t, .))_{\gamma}\right]_{\mu} \leq 2\left(\left[u_{0}\right]_{\mu}+\frac{C_{1}}{\gamma^{\frac{2-\mu}{2}}}\right) e^{\frac{\mu}{2} \bar{\gamma} t}} \\
& \quad \leq 2\left(\left[u_{0}\right]_{\mu}+\frac{\left(C_{R \vee R^{g}}^{F}+C^{F} R^{g}\right)}{\gamma^{\frac{2-\mu}{2}}}\right) e^{\frac{\mu}{2} \bar{\gamma} t}+\frac{2 C^{F} C^{g}}{\gamma^{\frac{2-\mu}{2}}} e^{\frac{\mu}{2} \bar{\gamma} t} \sup _{\tau \in[0, t]}\left[u_{\gamma}(\tau, .)\right]_{\mu} .
\end{aligned}
$$


Recalling that $\gamma>0$ is arbitrary, we can take $\gamma=\gamma_{1}(t)>0$ in this inequality, where $\gamma_{1}(t)$ is such that $\frac{2 C^{F} C^{g}}{\gamma_{1}(t)^{\frac{2-\mu}{2}}} e^{\frac{\mu}{2}} \bar{\gamma} t=\frac{1}{2}$. We then have proved that for all $u \in E^{\mu}$ and all $t \in[0, T]$

$$
\begin{aligned}
{\left[(\Theta u(t, .))_{\gamma_{1}(t)}\right]_{\mu} } & \leq 2\left(\left[u_{0}\right]_{\mu}+\frac{\left(C_{R \vee R^{g}}^{F}+C^{F} R^{g}\right)}{\gamma_{1}(t)^{\frac{2-\mu}{2}}}\right) e^{\frac{\mu}{2} \bar{\gamma} t}+\frac{1}{2} \sup _{\tau \in[0, t]}\left[u_{\gamma_{1}(t)}(\tau, .)\right]_{\infty}, \\
& \leq \frac{1}{2}\left(4\left[u_{0}\right]_{\mu} e^{\frac{\mu}{2} \bar{\gamma} t}+C_{R}\right)+\frac{1}{2} \sup _{\tau \in[0, t]}\left[u_{\gamma_{1}(t)}(\tau, .)\right]_{\infty},
\end{aligned}
$$

where $R$ is any $L^{\infty}$ bound on $E^{\mu}$ and $\gamma_{1}(t)$ and $C_{R}$ are defined as in Theorem 3.2. A simple computation now completes the proof of the stability of $E^{\mu}$ by $\Theta$.

Second step: conclusion. Since $E^{\mu}$ is a nonempty closed subspace of $E$, we deduce that the unique fixed point of $\Theta$ belongs to $E^{\mu}$. Thus, the unique viscosity solution $u$ of (1.1)-(1.2) is Hölder continuous. Noting now that (4.15) still holds true for $R=\max \left\{\|u\|_{\infty},\|\Theta u\|_{\infty}\right\}$ completes the proof of Theorem 3.2.

\subsection{Case of a coupled nonlocal term}

Proof of Theorem 3.3 We use a contracting fixed point theorem. For each $u \in$ $C_{b}\left(\bar{Q}_{T}\right)$, the Hamiltonian $F^{(u)}$ satisfies $(4.2)-(4.4)$ and $\sup \bar{Q}_{T}\left|F^{(u)}(t, x, 0,0,0)\right|<$ $+\infty$; hence, we can define $\Theta u$ as the unique continuous bounded viscosity solution of (2.2) which satisfies (1.2).

First step: a needed gradient estimate. Let us prove that for all $u \in C_{b}\left(\bar{Q}_{T}\right)$ and all $t \in[0, T]$,

$$
[\Theta u(t, .)]_{1} \leq\left(2\left[u_{0}\right]_{1}+C_{R} t\right) e^{\frac{\bar{\gamma}}{2} t},
$$

where $R=\max \left\{\|u\|_{\infty},\|\Theta u\|_{\infty}\right\}$ and $\bar{\gamma}$ and $C_{R}$ are defined as in Theorem 3.3.

Let us argue similarly as in the preceding proof. We now use the item i) of Theorem 4.1 for $G_{i}=F^{(u)}$ and $u_{i}=\Theta u(i=1,2)$. We get

$$
\Theta u(t, x)-(\Theta u)(t, y) \leq m_{\varepsilon}(0)+t \sup _{\tau \in[0, t]} \sigma_{\varepsilon}(\tau)+e^{\bar{\gamma} t} \frac{|x-y|^{2}}{2 \varepsilon} .
$$

Condition (H9) now gives the following estimate on $I$ (the quantity $I$ being defined for $r=0$ as in (4.12)): for all $\varepsilon>0$, all $t \in[0, T]$, all $\tau \in[0, t]$ and all $(x, y, r, p, X, Y) \in d_{\varepsilon}^{n}(\tau)$,

$$
\begin{aligned}
I \leq & C_{R \vee R^{g}}^{F}|x-y|\left(1+e^{\bar{\gamma} \tau} \frac{|x-y|}{\varepsilon}\right) \\
& +C^{F}|g[u](\tau, y)-g[u](\tau, x)|\left(1+e^{\bar{\gamma} \tau} \frac{|x-y|}{\varepsilon}+e^{\bar{\gamma} \tau} \frac{|g[u](\tau, y)-g[u](\tau, x)|}{\varepsilon}\right)
\end{aligned}
$$




$$
\begin{aligned}
\leq & C_{R \vee R^{g}}^{F}|x-y|\left(1+e^{\bar{\gamma} \tau} \frac{|x-y|}{\varepsilon}\right) \\
& +C^{F}[g[u]]_{1}|x-y|\left(1+e^{\bar{\gamma} \tau} \frac{|x-y|}{\varepsilon}+e^{\bar{\gamma} \tau} \frac{[g[u]]_{1}|x-y|}{\varepsilon}\right), \\
\leq & \left(C_{R \vee R^{g}}^{F}+C^{F}[g[u]]_{1}\right)|x-y|+\left(C_{R \vee R^{g}}^{F}+C^{F}\left[g[u]_{1}+C^{F}[g[u]]_{1}^{2}\right) e^{\bar{\gamma} \tau} \frac{|x-y|^{2}}{\varepsilon},\right.
\end{aligned}
$$

where $R:=\max \left\{\|u\|_{\infty},\|\Theta u\|_{\infty}\right\}$ and $R^{g}$ is defined as in Theorem 3.3. By (H2)", we get (4.13) for $\mu=1$ and $C_{1}$ which is now equal to $C_{R \vee R^{g}}^{F}+C^{F} R^{g}$. Inequalities (4.17), (4.11) and (4.14) and an optimization wrt $\in$ then complete the proof of (4.16).

Second step: local-in-time solvability of (1.1)-(1.2). For $\left.\left.t^{*} \in\right] 0, T\right]$, define the space

$$
E_{t^{*}}^{1}:=\left\{u \in C_{b}\left(\bar{Q}_{t^{*}}\right): u(0, .)=u_{0} \text { and (3.2) holds true for all } t \in\left[0, t^{*}\right]\right\}
$$

endowed with the distance of the uniform convergence. For each $u \in E_{t^{*}}^{1}$, we let $\Theta u$ also denote the unique bounded continuous viscosity solution of (1.1), in the domain $Q_{t^{*}}$, that satisfies (1.2). By Proposition 4.2, we known that $\Theta$ maps $E_{t^{*}}^{1}$ into itself. We leave it to the reader to verify that Proposition 4.2 still holds true under the assumptions of Theorem 3.3. Let us prove that $\Theta: E \rightarrow E$ is a contraction for $t^{*}$ sufficiently small.

In what follows, we let $R$ denote a $L^{\infty}$ bound on $E_{t^{*}}^{1}$ and we let $R^{\prime}$ denote a bound on $\Theta\left(E_{t^{*}}^{1}\right)$ for the $[\cdot]_{1}$-seminorm. Such a number $R^{\prime}$ exists, thanks to (4.16). Consider $u_{i} \in E_{t^{*}}^{1}(i=1,2)$. Define $N:=\sup _{t \in\left[0, t^{*}\right]}\left\{\Theta u_{1}(t,)-.\Theta u_{2}(t,).\right\}$ and $M:=\sup _{t \in\left[0, t^{*}\right]}\left\|u_{1}(t, .)-u_{2}(t, .)\right\|_{\infty}$. The item i) of Theorem 4.1, applied to the functions $\Theta u_{i}$, the Hamiltonians $F^{\left(u_{i}\right)}$ and $\bar{\gamma}=0$, implies that for all $\varepsilon>0$,

$$
N \leq m_{\varepsilon}(0)+t^{*} \sup _{t \in\left[0, t^{*}\right]} \sigma_{\varepsilon}(t) \leq \frac{\left[u_{0}\right]_{1}^{2}}{2} \varepsilon+t^{*} \sup _{t \in\left[0, t^{*}\right]} \sigma_{\varepsilon}(t) \leq \frac{R^{\prime 2}}{2} \varepsilon+t^{*} \sup _{t \in\left[0, t^{*}\right]} \sigma_{\varepsilon}(t) .
$$

Let us derive an upper bound on $\sup _{t \in\left[0, t^{*}\right]} \sigma_{\varepsilon}(t)$. Consider $t \in\left[0, t_{*}\right]$ and $(x, y, r, p$, $X, Y) \in d_{\varepsilon}^{n}(t)$. Condition (H9) implies that

$$
\begin{aligned}
& F^{\left(u_{2}\right)}(t, y, r, p, Y)-F^{\left(u_{1}\right)}(t, x, r, p, X) \leq \\
& \quad C_{R \vee R^{g}}^{F}|x-y|\left(1+\frac{|x-y|}{\varepsilon}\right)+C^{F}|\lambda-\mu|\left(1+\frac{|x-y|}{\varepsilon}+\frac{|\lambda-\mu|}{\varepsilon}\right),
\end{aligned}
$$

where $\lambda=g\left[u_{1}\right](t, y)$ and $\mu=g\left[u_{2}\right](t, x)$. By (H2)" and (H3),

$$
\begin{aligned}
|\lambda-\mu| & \leq\left[g\left[u_{1}\right](t, .)\right]_{1}|x-y|+\left|g\left[u_{1}\right](t, x)-g\left[u_{2}\right](t, x)\right|, \\
& \leq C^{g}\left(1+\sup _{\tau \in\left[0, t^{*}\right]}|| u_{1}(\tau, .) \mid \|_{\infty}\right)|x-y|+C^{g} M, \\
& \leq R^{g}|x-y|+C^{g} M .
\end{aligned}
$$


Using (4.19) and (4.20),

$$
\begin{aligned}
& F^{\left(u_{2}\right)}(t, y, r, p, Y)-F^{\left(u_{1}\right)}(t, x, r, p, X) \\
& \quad \leq C_{1}|x-y|\left(1+\frac{|x-y|}{\varepsilon}\right)+C_{2} M\left(1+\frac{|x-y|}{\varepsilon}+\frac{M}{\varepsilon}\right),
\end{aligned}
$$

where $C_{i}$ only depends on $C^{F}, C^{g}, C_{R \vee R^{g}}^{F}$ and $R(i=1,2)$. By the definition of $d_{\varepsilon}^{n}(t)$

$$
|x-y| \leq 2 \sqrt{\varepsilon} \sqrt{m_{2 \varepsilon}(t)-m_{\varepsilon}(t)+\frac{1}{n}} \leq 2 R^{\prime} \varepsilon+2 \sqrt{\varepsilon} \frac{1}{n} .
$$

Indeed if $m_{2 \varepsilon}(t)=0$, then (4.22) is immediate (since $x=y$ ). In the other case, that is to say if $m_{2 \varepsilon}(t)>0$, we can deduce from (4.37) that the approximate supremum $m_{2 \varepsilon, \eta}(t)$ (defined in (4.25)) is positive for all $\eta$ sufficiently small. If $m_{2 \varepsilon, \eta}(t)$ is achieved at some $(\bar{x}, \bar{y})$ (by $(4.24)$, such a maximal point always exists), then we see that $\frac{\bar{x}-\bar{y}}{2 \varepsilon} \in \partial^{1,+}\left(\Theta u_{2}(t,).\right)(\bar{y})$ and it follows that $|\bar{x}-\bar{y}| \leq 2 R^{\prime} \varepsilon$. By a simple computation, we get $m_{2 \varepsilon, \eta}(t)-m_{\varepsilon, \eta}(t) \leq \frac{|\bar{x}-\bar{y}|^{2}}{4 \varepsilon} \leq R^{\prime} \varepsilon$. The limit as $\eta \stackrel{\longrightarrow}{\longrightarrow} 0$ in this inequality then gives $m_{2 \varepsilon}(t)-m_{\varepsilon}(t) \leq R^{2} \varepsilon$. Inequality (4.22) is now a immediate consequence of the definition of $d_{\varepsilon}^{n}(t)$. Taking the supremum w.r.t. $t \in\left[0, t^{*}\right]$ and $(x, y, r, p, X, Y) \in d_{\varepsilon}^{n}(t)$ in (4.21) then gives

$$
\sup _{t \in\left[0, t^{*}\right]} \sigma_{\varepsilon}(t) \leq C_{3} \varepsilon+C_{4} M\left(1+\frac{M}{\varepsilon}\right),
$$

where $C_{i}$ only depends on $C^{F}, C^{g}, C_{R \vee R^{g}}^{F}, R$ and $R^{\prime}(i=3,4)$. Inequality (4.18) implies that for all $\varepsilon>0$,

$$
N \leq \frac{R \prime^{2}}{2} \varepsilon+t^{*}\left(C_{3} \varepsilon+C_{4} M\left(1+\frac{M}{\varepsilon}\right)\right)=t^{*} C_{4} M+\left(\frac{R \prime^{2}}{2}+t^{*} C_{3}\right) \varepsilon+t^{*} C_{4} \frac{M^{2}}{\varepsilon}
$$

By taking the infimum w.r.t. $\varepsilon$, there exists a universal constant $C \geq 0$ such that

$$
\sup _{t \in\left[0, t^{*}\right]}\left\{\Theta u_{1}(t, .)-\Theta u_{2}(t, .)\right\} \leq\left(t^{*} C_{4}+C\left(\frac{R^{\prime 2}}{2}+t^{*} C_{3}\right)^{\frac{1}{2}} \sqrt{t^{*}} \sqrt{C_{4}}\right) M .
$$

By exchanging the role of $\Theta u_{1}$ and $\Theta u_{2}$, we conclude that

$$
\begin{aligned}
\sup _{t \in\left[0, t^{*}\right]}\left\|\Theta u_{1}(t, .)-\Theta u_{2}(t, .)\right\|_{\infty} \leq \\
\left(t^{*} C_{4}+C\left(\frac{R \prime^{2}}{2}+t^{*} C_{3}\right)^{\frac{1}{2}} \sqrt{t^{*}} \sqrt{C_{4}}\right) \sup _{t \in\left[0, t^{*}\right]}\left\|u_{1}(t, .)-u_{2}(t, .)\right\|_{\infty} .
\end{aligned}
$$


Since $C_{i}$ only depends on $C^{F}, C^{g}, C_{R \vee R^{g}}^{F}, R$ and $R^{\prime}(i=3,4)$, we have proved that $\Theta$ is a contraction for $t^{*}>0$ sufficiently small. Consequently, there exists a unique $u^{t^{*}} \in C_{b}\left(\bar{Q}_{t^{*}}\right)$ such that $u^{t^{*}}$ is solution of (1.1)-(1.2) in $Q_{t^{*}}$.

Third step: global solvability. Define now

$$
\begin{aligned}
I:= & \{t \in] 0, T]: \text { there exists a unique } u^{t} \in C_{b}\left(\bar{Q}_{t}\right) \text { that satisfies (3.2) } \\
& \text { such that } \left.u^{t} \text { is solution of }(1.1)-(1.2) \text { in } Q_{t}\right\}
\end{aligned}
$$

and $t_{\max }=\sup I$. By proposition 4.2 and the preceding step, $I \neq \emptyset$. Let us prove that $t_{\max }=T$. Let us assume the contrary and let us seek a contradiction. By construction, there exists a unique $u^{t_{\max }} \in C_{b}\left(Q_{t_{\max }}\right)$ such that $u^{t_{\max }}$ is solution of (1.1)-(1.2) in $Q_{t_{\max }}$. By the gradient estimate of the first step (see (4.16)) and Proposition 4.1, $u^{t_{\max }}$ satisfies (3.2), (3.4) and (4.7). We leave it to the reader to verify that Proposition 4.1 still holds true under the assumptions of Theorem 3.3. The family $\left(u^{t_{\max }}(s, .)\right)_{s \in\left[0, t_{\max }[\right.}$ thus satisfies the Cauchy property for $s \leq t_{\max }$ and it follows that $u^{t_{\max }} \in C_{b}^{0,1}\left(\bar{Q}_{t_{\max }}\right)$. Consider the following Cauchy problem:

$$
\begin{aligned}
\partial_{t} u+F\left(t, x, u, D u, D^{2} u, \widetilde{g}[u]\right) & =0 \text { in }] t_{\max }, T\left[\times \mathbb{R}^{N},\right. \\
u\left(t_{\max }, .\right) & =u^{t_{\max }} \text { on } \mathbb{R}^{N}
\end{aligned}
$$

where $\widetilde{g}[u]:=g[w]$ with $w$ which is defined as follows: $w:=u^{t_{\max }}$ on $\bar{Q}_{t_{\max }}$ and $w=u$ on $\left[t_{\max }, t\right] \times \mathbb{R}^{N}$. Arguing as in the second step, we can prove that there exists $\left.t \in] t_{\max }, T\right]$ such that, there exists a unique $u \in C_{b}\left(\left[t_{\text {max }}, t\right] \times \mathbb{R}^{N}\right)$ solution of (4.23) in $] t_{\text {max }}, t\left[\times \mathbb{R}^{N}\right.$. For each $u \in C_{b}\left(\left[t_{\text {max }}, t\right] \times \mathbb{R}^{N}\right)$ which satisfies $u\left(t_{\max },.\right)=u^{t_{\max }}\left(t_{\max },.\right)$, define $\widetilde{u} \in C_{b}\left(\bar{Q}_{t}\right)$ as follows: $\widetilde{u}=u^{t_{\max }}$ on $\bar{Q}_{t_{\max }}$ and $\widetilde{u}=u$ on $\left[t_{\max }, t\right] \times \mathbb{R}^{N}$. Since

$$
\mathcal{P}^{2,(+)-} \widetilde{u}\left(t_{\max }, x\right) \subseteq \mathcal{P}_{\bar{Q}_{t_{\max }}^{2,(+)-}}^{t_{\max }}\left(t_{\max }, x\right),
$$

the function $u$ is solution of (4.23) in $] t_{\max }, t\left[\times \mathbb{R}^{N}\right.$ iff $\widetilde{u}$ is solution of (1.1) in $Q_{t}$. We deduce that $t \in I$. Since $t>t_{\max }$, we get a contradiction and necessarily $t_{\max }=T$. The proof of Theorem 3.3 is now complete (note that (4.16) implies $(3.4))$.

\subsection{Proof of Theorem 4.1}

Before proving our continuous dependence estimate, we have to recall two classical lemmas. The first lemma establishes a relation between the Fréchet subdifferential and the Clarke generalized derivative of a function of the real variable and the second lemma is a semicontinuity result on marginal functions.

Here is the first result. 
Lemma 4.1 Let $f:[0, T] \rightarrow \mathbb{R}$ be a locally bounded function. For each $s \in] 0, T[$,

$$
\sup \partial^{1,+} f(s) \leq \limsup _{\substack{t \rightarrow s \\ h \geq 0}} \frac{f(t+h)-f(t)}{h} .
$$

Proof. Let $\xi$ belong to $\partial^{1,+} f(s)$. For each $\tau$ sufficiently small, $f(s+\tau) \leq f(s)+$ $\xi \tau+o(\tau)$. If $\tau<0$, then

$$
\xi \leq \frac{f(s+\tau)-f(s)}{\tau}+\frac{o(\tau)}{\tau}=\frac{f(t)-f(t+h)}{-h}+\frac{o(h)}{h},
$$

where $t+h:=s$ and $t:=s+\tau$. Since $t \rightarrow s$ and $h \stackrel{>}{\rightarrow}$ when $\tau \stackrel{\leq}{\rightarrow}$, the proof of Lemma 4.1 is complete.

To state the second lemma, we have to introduce some notations and recall some definitions on multiapplications. Consider $E$ and $F$ two metric spaces, $k$ an integer, and $f$ a given function from $E \times F$ into $\mathbb{R}$. For $x \in E$, define $g(x):=$ $\sup _{y \in d(x)} f(x, y)$ where we let $d(x)$ denote any subset of $F$ depending on $x$.

Definition 4.1 The multiapplication $d: E \rightrightarrows F$ is said to be nonempty valued if for each $x \in E, d(x)$ is a nonempty subset of $F$. The multiapplication $d$ is said to be

i) upper semicontinuous on $E$ (u.s.c. for short) iff for all $x \in E$ and all neighbourhood $\mu$ of $d(x)$, there exists $\eta>0$ such that for all $x^{\prime} \in \bar{B}_{E}(x, \eta)$ $d\left(x^{\prime}\right) \subseteq \mu\left({ }^{1}\right)$

ii) lower semicontinuous on $E$ (l.s.c. for short) iff for all $x \in E$, all sequence $x_{m} \rightarrow x$ and all $y \in d(x)$, there exists a sequence $y_{m} \in d\left(x_{m}\right)$ such that $y_{m} \rightarrow y$

iii) continuous on $E$ iff it is both u.s.c. and l.s.c. on $E$.

Here is the second lemma.

Lemma 4.2 Assume that $f$ is continuous and that $d$ is nonempty valued, u.s.c. on $E$ and such that $\operatorname{Im}(d):=\cup_{x \in E} d(x)$ is relatively compact. Then, $g: E \rightarrow \mathbb{R}$ is well-defined and upper semicontinuous. If moreover $d$ is l.s.c. on $E$, then $g$ is continuous on $E$.

\footnotetext{
${ }^{1}$ In this paper, we only consider multiapplications of the form $d(x)=\{y \in F: h(x, y) \leq 0\}$, where $h: E \times F \rightarrow \mathbb{R}^{k}$ is continuous and $\operatorname{Im}(d):=\cup_{x \in E} d(x)$ is relatively compact; note that such multiapplications are always u.s.c.
} 
For a proof of this result we refer the reader to [5, Theorem 7.3.1]. Let us now return to the proof of our continuous dependence estimate.

Proof of Theorem 4.1 Let us introduce some notations that will be needed. Define

$$
\psi_{\varepsilon}(t, x, y):=u_{1}(t, x)-u_{2}(t, y)-e^{\bar{\gamma} t} \frac{|x-y|^{2}}{2 \varepsilon},
$$

and

$$
\begin{aligned}
& \Psi\left(t, x, y, r_{1}, r_{2}, p, X, Y\right):= \\
& \quad\left(G_{2}\left(t, y, r_{2}, e^{\bar{\gamma} t} p, e^{\bar{\gamma} t} Y\right)-G_{1}\left(t, x, r_{1}, e^{\bar{\gamma} t} p, e^{\bar{\gamma} t} X\right)-\bar{\gamma} e^{\bar{\gamma} t} \frac{|x-y|^{2}}{2 \varepsilon}\right)^{+} .
\end{aligned}
$$

Let us perturb the functions $m_{\varepsilon}($.$) and \sigma_{\varepsilon}($.$) the following way: let \phi \in C^{2}\left(\mathbb{R}^{N}\right)$ be nonnegative and such that $\phi(0)=0, C^{\phi}:=\|D \phi\|_{\infty}+\left\|D^{2} \phi\right\|_{\infty}<+\infty$ and

$$
\lim _{|x| \rightarrow+\infty} \phi(x)=+\infty
$$

for $\eta \in] 0,1]$ and $t \in[0, T]$, define $\left({ }^{2}\right)$

$$
\begin{aligned}
m_{\varepsilon, \eta}(t) & :=\sup _{(x, y) \in \mathbb{R}^{N} \times \mathbb{R}^{N}}\left(\psi_{\varepsilon}(t, x, y)-\eta(\phi(x)+\phi(y))\right)^{+}, \\
\sigma_{\varepsilon, \eta}(t) & :=\sup _{d_{\varepsilon, \eta}(t)} \Psi(t, x, y, r, r, p, X, Y),
\end{aligned}
$$

where we let $d_{\varepsilon, \eta}(t)$ denote the set of $(x, y, r, p, X, Y)$ such that,

$$
\left\{\begin{array}{l}
\phi(x)+\phi(y) \leq \frac{2\left(\left\|u_{1}\right\|_{\infty} \vee\left\|u_{2}\right\|_{\infty}\right)}{\eta} \\
|r| \leq \max _{i=1,2} \sup _{\left\{\phi \leq \frac{2\left(\left\|u_{1}\right\|\left\|_{\infty} \vee\right\| u_{2}\|\|_{\infty}\right)}{\eta}\right\}}\left|u_{i}(t, .)\right| \\
p=\frac{x-y}{\varepsilon} \\
\text { Condition (3.1) holds true } \\
\frac{|x-y|^{2}}{4 \varepsilon} \leq m_{2 \varepsilon, \eta}(t)-m_{\varepsilon, \eta}(t)
\end{array}\right.
$$

Let us give some properties on these functions. We admit the result below for a while until the end of the proof of Theorem 4.1.

Proposition 4.3 The functions $m_{\varepsilon, \eta}($.$) and \sigma_{\varepsilon, \eta}($.$) are well-defined, real valued$ and continuous on $[0, T]$.

\footnotetext{
${ }^{2}$ The presence of the penalization term $\eta \phi(y)$ is not necessary, but it will simplify the rest of the proof.
} 
The strategy of the proof of Theorem 4.1 is the following: first, we will prove that there exists a constant $R \geq 0$ such that for each $\eta \in] 0,1]$,

$m_{\varepsilon, \eta}($.$) is a continuous viscosity subsolution of the following$

differential equation in $f: \dot{f}=\left(\sigma_{\varepsilon, \eta}-\gamma f\right)^{+}+2 \omega_{R}\left(\eta C^{\phi}\right)$ in $] 0, T[$,

where we let $\omega_{R}($.$) denote the modulus deriving from (4.2); this will give us$ some approximate continuous dependence estimates, thanks to the comparison principle, and we will conclude by taking the limit as $\eta \stackrel{\gg}{\rightarrow}$ in these estimates.

First step: proof of (4.26). By Proposition 4.3 and Lemma 4.1, (4.26) can be seen as a consequence of the following property: for each $s \in] 0, T[$,

$$
\limsup _{\substack{t \rightarrow s \\ h \gtrsim 0}} \frac{m_{\varepsilon, \eta}(t+h)-m_{\varepsilon, \eta}(t)}{h} \leq\left(\sigma_{\varepsilon, \eta}(s)-\gamma m_{\varepsilon, \eta}(s)\right)^{+}+2 \omega_{R}\left(\eta C^{\phi}\right) .
$$

To prove (4.27), we have to perturb again the functions $m_{\varepsilon, \eta}($.$) and \sigma_{\varepsilon, \eta}($.$) the$ following way: define

$$
E:=\left\{(t, h) \in \mathbb{R}^{2}: 0 \leq t \leq t+h \leq T\right\}
$$

and for every $(t, h) \in E$ and $\lambda \in \mathbb{R}^{+}$, define

$$
\begin{aligned}
M_{\varepsilon}(t, h, \lambda):= & \sup _{\substack{\tau \in[t, t+h],(x, y) \in \mathbb{R}^{N} \times \mathbb{R}^{N}}}\left(\psi_{\varepsilon}(\tau, x, y)-\eta(\phi(x)+\phi(y))-\lambda(\tau-t)\right)^{+}, \\
\Sigma(t, h, \lambda):= & \sup _{D(t, h, \lambda)} \Psi\left(\tau, x, y, r_{1}, r_{2}, p, X, Y\right),
\end{aligned}
$$

where we let $D(t, h, \lambda)$ denote the set of $\left(\tau, x, y, r_{1}, r_{2}, p, X, Y\right)$ such that,

$$
\left\{\begin{array}{l}
\tau \in[t, t+h] \\
\phi(x)+\phi(y) \leq \frac{2\left(\left\|u_{1}\right\|_{\infty} \vee\left\|u_{2}\right\|_{\infty}\right)}{\eta} \\
r_{1}-r_{2} \geq M_{\varepsilon}(t, h, \lambda), \\
\left|r_{i}\right| \leq \max _{j=1,2} \sup _{[t, t+h] \times\left\{\phi \leq \frac{2\left(\left\|u_{1}\right\|_{\infty} \vee\left\|u_{2}\right\|_{\infty}\right)}{\eta}\right\}}\left|u_{j}\right|, \quad(i=1,2) \\
p=\frac{x-y}{\varepsilon} \\
\text { Condition }(3.1) \text { holds true, } \\
\frac{|x-y|^{2}}{4 \varepsilon} \leq M_{2 \varepsilon}(t, h, \lambda)-M_{\varepsilon}(t, h, \lambda)
\end{array}\right.
$$

Here are some properties on these functions, that we admit until the end of the proof of Theorem 4.1.

Proposition 4.4 The functions $M_{\varepsilon}(., . .$.$) and \Sigma(., .,$.$) are well-defined, real val-$ ued and continuous on $E \times \mathbb{R}^{+}$. 
The new domain, on which the supremum $M_{\varepsilon}(t, h, \lambda)$ is computed, is introduced in order to use Equation (4.1). Note the presence of the penalization terms, $\eta(\phi(x)+\phi(y))$ and $\frac{|x-y|^{2}}{2 \varepsilon}$, which are used classically when working with viscosity solutions (see [9]). Let us recall that there are respectively used to treat unbounded domain and to split in two the space variables and use Ishii Lemma.

The strategy of the proof of (4.27) is the following: for every $(t, h) \in E$, the parameter $\lambda$ will be chosen large enough in order to ensure that

$$
M_{\varepsilon}(t, h, \lambda) \leq M_{\varepsilon}(t, 0, \lambda)=m_{\varepsilon, \eta}(t)
$$

hence, we will infer that $\frac{m_{\varepsilon, \eta}(t+h)-m_{\varepsilon, \eta}(t)}{h} \leq \lambda$ and (4.27) will be proved by passing to the limit in this inequality as $t \rightarrow s$ and $h \stackrel{>}{\rightarrow}$.

Construction of the best $\lambda$ satisfying (4.28). Let us take

$$
R=\max \left\{\left\|u_{1}\right\|_{\infty} \vee\left\|u_{2}\right\|_{\infty}, 2 \sqrt{\frac{2\left(\left\|u_{1}\right\|_{\infty} \vee\left\|u_{2}\right\|_{\infty}\right) e^{\bar{\gamma} T}}{\varepsilon}}+C^{\phi}, \frac{6 e^{\bar{\gamma} T}}{\varepsilon}+C^{\phi}\right\} .
$$

For every $(t, h) \in E$, define

$$
\lambda(t, h):=\sup d(t, h)
$$

where we let $d(t, h)$ denote the set of $\lambda \in \mathbb{R}^{+}$such that

$$
\lambda-\Sigma(t, h, \lambda)-2 \omega_{R}\left(\eta C^{\phi}\right) \leq 0 .
$$

We have the following result:

Proposition 4.5 The function $\lambda(.,$.$) is well-defined, real valued and u.s.c on E.$

Proof. It is easy to see that $\Sigma(t, h, \lambda)$ is nonnegative and bounded independently of $(t, h, \lambda) \in E \times \mathbb{R}^{+}$. Then, $d(.,$.$) is nonempty valued and \cup_{(t, h) \in E} d(t, h)$ is relatively compact. The continuity of $\Sigma(., .,$.$) (see proposition 4.4$ ) implies that the multiapplication $d(.,$.$) is u.s.c. on E$ (see footnote 1 on page 17 ). Lemma 4.2 then completes the proof of Proposition 4.5.

Let us prove that for all $(t, h) \in E$,

$$
M_{\varepsilon}(t, h, \lambda(t, h)) \leq M_{\varepsilon}(t, 0, \lambda(t, h)) .
$$

When $M_{\varepsilon}(t, h, \lambda(t, h))=0,(4.32)$ is immediate (since $M_{\varepsilon}(., .,$.$) is nonnegative).$ Assume that $M_{\varepsilon}(t, h, \lambda(t, h))>0$. The continuity $M_{\varepsilon}(., .,$.$) (see Proposition 4.4)$ implies that for all $\lambda>\lambda(t, h)$ sufficiently close to $\lambda(t, h), M_{\varepsilon}(t, h, \lambda)>0$. Let $\lambda$ 
be such a number. By Condition (4.24), there exists $(\bar{\tau}, \bar{x}, \bar{y})$ at which $M_{\varepsilon}(t, h, \lambda)$ is achieved. Necessarily,

$$
\left\{\begin{array}{l}
\phi(\bar{x})+\phi(\bar{y}) \leq \frac{2\left(\left\|u_{1}\right\|_{\infty} V\left\|\mid u_{2}\right\|_{\infty}\right)}{\eta}, \\
e^{\bar{\gamma} \tau} \frac{\bar{x}-\left.\bar{y}\right|^{2}}{4 \varepsilon} \leq M_{2 \varepsilon}(t, h, \lambda)-M_{\varepsilon}(t, h, \lambda) .
\end{array}\right.
$$

Let us prove that $\bar{\tau}=t$. Assume the contrary and let us seek a contradiction. We let $\mathcal{O}$ denote the cylinder $] t, t+h] \times \mathbb{R}^{N}$. Ishii Lemma (see [8] or [9, Theorem 8.3]) implies that there are

$$
\begin{aligned}
\left(a, e^{\overline{\gamma \tau}} \bar{p}+\eta D \phi(\bar{x}), e^{\overline{\gamma \tau}} X+\eta D^{2} \phi(\bar{x})\right) & \in \overline{\mathcal{P}}_{\mathcal{O}}^{2,+} u_{1}(\bar{\tau}, \bar{x}), \\
\left(b, e^{\overline{\gamma \tau}} \bar{p}-\eta D \phi(\bar{y}), e^{\overline{\gamma \tau}} Y-\eta D^{2} \phi(\bar{y})\right) & \in \overline{\mathcal{P}}_{\mathcal{O}}^{2,-} u_{2}(\bar{\tau}, \bar{y}),
\end{aligned}
$$

with

$$
\left\{\begin{array}{l}
\bar{p}=\frac{\bar{x}-\bar{y}}{\varepsilon} \\
a-b=\lambda+\bar{\gamma} e^{\overline{\gamma \tau} \tau} \frac{|\bar{x}-\bar{y}|^{2}}{2 \varepsilon} \\
\text { Condition (3.1) holds true }
\end{array}\right.
$$

Using the viscosity inequalities, we find that

$$
\begin{aligned}
a-b \leq G_{2}\left(\bar{\tau}, \bar{y}, u_{2}, e^{\overline{\gamma \tau}} \bar{p}-\right. & \left.\eta D \phi(\bar{y}), e^{\overline{\gamma \tau}} Y-\eta D^{2} \phi(\bar{y})\right) \\
& -G_{1}\left(\bar{\tau}, \bar{x}, u_{1}, e^{\overline{\gamma \tau}} \bar{p}+\eta D \phi(\bar{x}), e^{\overline{\gamma \tau}} X+\eta D^{2} \phi(\bar{x})\right) .
\end{aligned}
$$

By (4.33) and (3.1), we know that $\left|e^{\overline{\gamma \tau}} \bar{p}\right| \leq 2 \sqrt{\frac{2\left(\left\|u_{1}\right\|_{\infty} V\left\|\mid u_{2}\right\| \|_{\infty}\right) e^{\overline{\gamma \tau}}}{\varepsilon}}$ and $|X|,|Y| \leq$ $\frac{6}{\varepsilon}$. Recalling that $\bar{\gamma}$ is nonnegative, the definition of $R$ (see (4.29)) and (4.2) imply that

$$
a-b \leq G_{2}\left(\bar{\tau}, \bar{y}, u_{2}, e^{\overline{\gamma \tau}} \bar{p}, e^{\bar{\gamma} \bar{\tau}} Y\right)-G_{1}\left(\bar{\tau}, \bar{x}, u_{1}, e^{\overline{\gamma \tau}} \bar{p}, e^{\overline{\gamma \tau}} X\right)+2 \omega_{R}\left(\eta C^{\phi}\right) .
$$

We then deduce that

$$
\begin{aligned}
\lambda \leq & G_{2}\left(\bar{\tau}, \bar{y}, u_{2}, e^{\overline{\gamma \tau}} \bar{p}, e^{\overline{\gamma \tau}} Y\right)-G_{1}\left(\bar{\tau}, \bar{x}, u_{1}, e^{\overline{\gamma \tau}} \bar{p}, e^{\overline{\gamma \tau}} X\right) \\
& -\bar{\gamma} e^{\overline{\gamma \tau}} \frac{|\bar{x}-\bar{y}|^{2}}{2 \varepsilon}+2 \omega_{R}\left(\eta C^{\phi}\right), \\
\leq & \Psi\left(\bar{\tau}, \bar{x}, \bar{y}, r_{1}, r_{2}, \bar{p}, X, Y\right)+2 \omega_{R}\left(\eta C^{\phi}\right),
\end{aligned}
$$

where $r_{1}=u_{1}(\bar{\tau}, \bar{x})$ and $r_{2}=u_{2}(\bar{\tau}, \bar{y})$. Recalling that $M_{\varepsilon}(t, h, \lambda)>0$, we get

$$
\left(\psi_{\varepsilon}(\bar{\tau}, \bar{x}, \bar{y})-\eta(\phi(\bar{x})+\phi(\bar{y}))-\lambda(\bar{\tau}-t)\right)^{+}>0 .
$$

As a result of this,

$$
\begin{aligned}
\psi_{\varepsilon}(\bar{\tau}, \bar{x}, \bar{y})-\eta(\phi(\bar{x})+\phi(\bar{y}))-\lambda(\bar{\tau}-t) & = \\
& \left(\psi_{\varepsilon}(\bar{\tau}, \bar{x}, \bar{y})-\eta(\phi(\bar{x})+\phi(\bar{y}))-\lambda(\bar{\tau}-t)\right)^{+}=M_{\varepsilon}(t, h, \lambda) .
\end{aligned}
$$




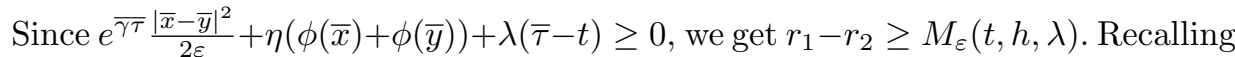
that (4.33) and (3.1) hold true, $\left(\bar{\tau}, \bar{x}, \bar{y}, r_{1}, r_{2}, \bar{p}, X, Y\right)$ belongs to $D(t, h, \lambda)$. By (4.34), $\lambda \leq \Sigma(t, h, \lambda)+2 \omega_{R}\left(\eta C^{\phi}\right)$. By (4.30), (4.31) and the fact that $\lambda>\lambda(t, h)$, we get a contradiction. Consequently, $\bar{\tau}=t$ and $M_{\varepsilon}(t, h, \lambda) \leq M_{\varepsilon}(t, 0, \lambda)$. By the continuity of $M_{\varepsilon}(., .,$.$) , we can pass to the limit in \lambda \stackrel{\gg}{\rightarrow} \lambda(t, h)$ to complete the proof of (4.32).

Passing to the limit as $t \rightarrow s$ and $h \stackrel{>}{\rightarrow}$. By a simple computation, we deduce from (4.32) that for all $(t, h) \in E$,

$$
\begin{aligned}
m_{\varepsilon, \eta}(t+h) & \leq M_{\varepsilon}(t, h, \lambda(t, h))+\lambda(t, h) h \\
& \leq M_{\varepsilon}(t, 0, \lambda(t, h))+\lambda(t, h) h \\
& =m_{\varepsilon, \eta}(t)+\lambda(t, h) h .
\end{aligned}
$$

It follows that for all $s \in] 0, T[$,

$$
\limsup _{\substack{t \rightarrow s \\ h \rightarrow 0}} \frac{m_{\varepsilon, \eta}(t+h)-m_{\varepsilon, \eta}(t)}{h} \leq \limsup _{\substack{t \rightarrow s \\ h}} \lambda(t, h) \leq \lambda(s, 0),
$$

thanks to the upper semicontinuity of $\lambda(.,$.$) (see Proposition 4.5). But, the con-$ tinuity of $\Sigma(., .,$.$) and the definition of \lambda(.,$.$) imply that$

$$
\lambda(s, 0)=\Sigma(s, 0, \lambda(s, 0))+2 \omega_{R}\left(\eta C^{\phi}\right)
$$

(in fact, this holds true not only for $(t, h)=(s, 0)$ but for all $(t, h) \in E$ ). Moreover, (4.3) implies that

$$
\Sigma(s, 0, \lambda(s, 0)) \leq\left(\sigma_{\varepsilon, \eta}(s)-\gamma M_{\varepsilon}(s, 0, \lambda(s, 0))\right)^{+}=\left(\sigma_{\varepsilon, \eta}(s)-\gamma m_{\varepsilon, \eta}(s)\right)^{+} .
$$

We deduce that $\lambda(s, 0) \leq\left(\sigma_{\varepsilon, \eta}(s)-\gamma m_{\varepsilon, \eta}(s)\right)^{+}+2 \omega_{R}\left(\eta C^{\phi}\right)$. This implies $(4.27)$ and this thus completes the proof of (4.26).

Second step: taking limit as $\eta \stackrel{\gg}{\rightarrow}$. By the comparison principle, we deduce from (4.26) that for each $t \in[0, T]$,

$$
m_{\varepsilon, \eta}(t) \leq m_{\varepsilon, \eta}(0)+\int_{0}^{t} \sigma_{\varepsilon, \eta}(\tau) d \tau+2 t \omega_{R}\left(\eta C^{\phi}\right) ;
$$

moreover, if $\gamma>0$, then for each $t \in[0, T]$

$$
m_{\varepsilon, \eta}(t) \leq m_{\varepsilon, \eta}(0)+\frac{1}{\gamma} \sup _{\tau \in[0, t]} \sigma_{\varepsilon, \eta}(\tau) d \tau+2 t \omega_{R}\left(\eta C^{\phi}\right)
$$

Indeed, the right hand side of (4.35) (resp. (4.36)) is a classical solution (resp. a continuous supersolution) of (4.26) as function of the $t$-variable. Let us pass to the limit as $\eta \stackrel{\gg}{\longrightarrow} 0$ in these inequalities. 
For all $t \in[0, T]$,

$$
\lim _{\eta \geq 0} m_{i \varepsilon, \eta}(t)=m_{i \varepsilon}(t) \quad(i=1,2) \quad \text { and } \limsup _{\eta \rightarrow 0} \sigma_{\varepsilon, \eta}(t) \leq \sigma_{\varepsilon}(t) .
$$

Indeed, let $n$ be a nonnegative integer and let $t$ belong to $[0, T]$. For all positive $\eta$ sufficiently small, $m_{2 \varepsilon, \eta}(t)-m_{\varepsilon, \eta}(t) \leq m_{2 \varepsilon}(t)-m_{\varepsilon}(t)+\frac{1}{n}$. Consequently, $d_{\varepsilon, \eta}(t) \subseteq d_{\varepsilon}^{n}(t)$ and $\sigma_{\varepsilon, \eta}(t) \leq \sigma_{\varepsilon}^{n}(t)$. Taking the infimum w.r.t. $n \in \mathbb{N}^{*}$ implies that $\lim \sup _{\eta \geq 0} \sigma_{\varepsilon, \eta}(t) \leq \sigma_{\varepsilon}(t)$. The proof of the other limits of (4.37) is left to the reader. The set $d_{\varepsilon, \eta}(t)$ is bounded independently of $t$ and $\eta$. We deduce, by (4.4) and (4.5), that the family of functions $\left(\sigma_{\varepsilon, \eta}\right)_{\eta>0}$ is uniformly bounded on $[0, T]$. By (4.37) and Fatou's Lemma, the limit as $\eta \rightrightarrows 0$ in (4.35) completes the proof of the item i) of Theorem 4.1 (for a proof of the measurability of $\sigma_{\varepsilon}($.$) , see$ Appendix B).

If $\gamma>0$, then $U_{\tau \in[0, t]}\{r\}+d_{\varepsilon, \eta}(t) \subseteq D_{\varepsilon}(t)$. Thus, the item ii) of Theorem 4.1 can easily be deduced from (4.36). This completes the proof of Theorem 4.1.

Proof of Propositions 4.3 and 4.4 We only prove the continuity of $\Sigma(., . .$.$) since$ the other proofs are easier or very similar. Let us use Lemma 4.2, with $f=\Psi$ and $d()=.D(., .,$.$) . It is immediate that \Psi$ is continuous and we leave to the reader to verify that the reader verify that $\cup_{(t, h, \lambda) \in E \times \mathbb{R}^{+}} D(t, h, \lambda)$ is relatively compact and that $D(., .,$.$) is u.s.c.. Note that for all (t, h, \lambda) \in E \times \mathbb{R}^{+}$,

$$
\left(t, 0,0, r^{\infty},-r^{\infty}, 0,0,0\right) \in D(t, h, \lambda)
$$

where $r^{\infty}$ is defined as in (4.40). This proves that $D(., .,$.$) is nonempty valued. Let$ us prove that $D(., .,$.$) is l.s.c.. Let (t, h, \lambda)$ belong to $E \times \mathbb{R}^{+},\left(t_{m}, h_{m}, \lambda_{m}\right)_{n \in \mathbb{N}^{*}} \in$ $\left(E \times \mathbb{R}^{+}\right)^{\mathbb{N}^{*}}$ be a sequence which converges to $(t, h, \lambda)$ and $\left(\tau, x, y, r_{1}, r_{2}, p, X, Y\right)$ belong to $D(t, h, \lambda)$. For $m \in \mathbb{N}^{*}$, define

$$
\begin{aligned}
r_{m}^{\infty} & :=\max _{j=1,2} \sup _{\left[t_{m}, t_{m}+h_{m}\right] \times\left\{\phi \leq \frac{2\left(\left.\left\|u_{1}\right\|\right|_{\infty} v\left\|u_{2}\right\| \|_{\infty}\right)}{\eta}\right\}}\left|u_{j}\right|, \\
M_{m} & :=M_{\varepsilon}\left(t_{m}, h_{m}, \lambda_{m}\right)
\end{aligned}
$$

and

$$
\begin{aligned}
\tau_{m} & :=\max \left\{t_{m}, \min \left\{\tau, t_{m}+h_{m}\right\}\right\} \\
r_{1, m} & :=\max \left\{-r_{m}^{\infty}, \min \left\{M_{m}, r_{1}, r_{m}^{\infty}\right\}\right\} \\
r_{2, m} & :=\max \left\{-r_{m}^{\infty}, \min \left\{r_{2}, r_{1, m}-M^{m}\right\}\right\} .
\end{aligned}
$$

For any $\nu \in[0,1]$, define $y_{\nu}:=(1-\nu) x+\nu y$. We get $\frac{\left|x-y_{\nu}\right|^{2}}{4 \varepsilon}=\nu^{2} \frac{|x-y|^{2}}{4 \varepsilon}$. Define

$$
\nu_{m}^{2}:=\min \left\{1, \frac{4 \varepsilon}{|x-y|^{2}}\left(M_{2 \varepsilon}\left(t_{m}, h_{m}, \lambda_{m}\right)-M_{\varepsilon}\left(t_{m}, h_{m}, \lambda_{m}\right)\right)\right\}
$$


and $y_{m}=y_{\nu_{m}}$ and $p_{m}=\frac{x-y_{m}}{\varepsilon}$. Let us prove that

$$
\left(\tau_{m}, x, y_{m}, r_{1, m}, r_{2, m}, p_{m}, X, Y\right) \in D\left(t_{m}, h_{m}, \lambda_{m}\right)
$$

and

$$
\left(\tau_{m}, x, y_{m}, r_{1, m}, r_{2, m}, p_{m}, X, Y\right) \rightarrow\left(\tau, x, y, r_{1}, r_{2}, p, X, Y\right) .
$$

We only verify that $r_{i, m} \rightarrow r_{i}(\mathrm{i}=1,2)$, which is the most difficult point to prove. Let us first verify (4.38). We only verify the conditions on $r_{i, m}(i=1,2)$ and we leave the verification of the other conditions to the reader. That is to say, we have to verify that $\left|r_{i, m}\right| \leq r_{m}^{\infty}(i=1,2)$ and that $r_{1, m}-r_{2, m} \geq M_{n}$. Observe that for all reals $a \leq b$ and $c$,

$$
a \leq \max \{a, \min \{c, b\}\} \leq b \text { and }
$$

$$
\text { if } a \leq c \leq b \text {, then } \max \{a, \min \{c, b\}\}=c \text {. }
$$

Since $M_{m} \leq 2 r_{m}^{\infty}$, we see that $-r_{m}^{\infty}+M_{m} \leq r_{m}^{\infty}$. By (4.39) and the nonnegativity of $M_{m}$, we infer that $-r_{m}^{\infty} \leq-r_{m}^{\infty}+M_{m} \leq r_{1, m} \leq r_{m}^{\infty}$. Using now that $-r_{m}^{\infty} \leq$ $r_{1, m}-M_{m}$, (4.39) implies that $-r_{m}^{\infty} \leq r_{2, m} \leq r_{1, m}-M_{m} \leq r_{m}^{\infty}-M_{m} \leq r_{m}^{\infty}$. We then conclude that (4.38) holds true.

Moreover, we have the following properties:

$$
\begin{aligned}
& r_{m}^{\infty} \rightarrow r^{\infty}:=\max _{j=1,2} \sup _{[t, t+h] \times\left\{\phi \leq \frac{2\left(\left\|u_{1}\right\| \|\left._{\infty} \vee\left|u_{2}\right|\right|_{\infty}\right)}{\eta}\right\}}\left|u_{j}\right|, \\
& M_{m} \rightarrow M:=M_{\varepsilon}(t, h, \lambda) .
\end{aligned}
$$

This can be seen by using Lemma 4.2 to prove the continuity of $r^{\infty}$ and $M$, as functions of the $(t, h)$-variable. By the continuity of the functions $\min \{.,$.$\} and$ $\max \{.,$.$\} , we get$

$$
\begin{aligned}
& r_{1, m} \rightarrow \max \left\{-r^{\infty}+M, r_{1}, r^{\infty}\right\}, \\
& r_{2, m} \rightarrow \max \left\{-r^{\infty}, \min \left\{r_{2}, \lim _{m} r_{1, m}-M\right\}\right\} .
\end{aligned}
$$

Since $\left|r_{i}\right| \leq r^{\infty}$ and $r_{1}-r_{2} \geq M$ (because $\left.\left(\tau, x, y, r_{1}, r_{2}, p, X, Y\right) \in D(t, h, \lambda)\right)$, we deduce that $-r^{\infty}+M \leq r_{2}+M \leq r_{1} \leq r^{\infty}$ and (4.39) implies that $\lim _{m} r_{1, m}=r_{1}$ and $\lim _{m} r_{2, m}=r_{2}$. The proof of Propositions 4.3 and 4.4 is now complete.

\section{A Sketch of the proof of Proposition 4.1}

Let

$$
\begin{aligned}
C_{\nu}^{\prime} & :=\sup _{\beta>0} \frac{\sup _{t \in[0, T]} \omega_{\beta}(\Theta u(t, .))-\nu}{\beta^{2}}, \\
M & :=2 \sqrt{\frac{2 R}{C_{\nu}^{\prime}}} \\
C_{\nu} & :=\sup _{K}|F(t, x, 0, p, X, \lambda)|,
\end{aligned}
$$


where $R=\|u\|_{\infty} \vee\|\Theta u\|_{\infty}$ and $K$ denote the set of $(t, x, p, X, \lambda)$ such that,

$$
\left\{\begin{array}{l}
t \in[0, T] \\
|p| \leq C_{\nu}^{\prime} M \\
|X| \leq C_{\nu}^{\prime} \\
|\lambda| \leq C^{g}(1+R)
\end{array}\right.
$$

By (H4), (H7) and (H8), we know that $C_{\nu}$ is finite. Let $s$ and $y$ be fixed. A simple computation shows that the function

$$
v(t, x):=\Theta u(s, y)+\nu+C_{\nu}^{\prime} \frac{|x-y|^{2}}{2}+C_{\nu}(t-s)
$$

is a viscosity subsolution of $(2.2)$ in $] s, T\left[\times B_{\mathbb{R}^{N}}(y, M)\right.$. Moreover, for each $x$

$$
\Theta u(s, x)-\Theta u(s, y) \leq \omega_{|x-y|}(\Theta u(s, .)) \leq \nu+C_{\nu}^{\prime} \frac{|x-y|^{2}}{2} .
$$

It follows that $\Theta u(s,.) \leq v(s,$.$) . Another simple computation shows that \Theta u \leq v$ on the domain $] s, T\left[\times \partial \bar{B}_{\mathbb{R}^{N}}(y, M)\right.$. Using the comparison principle with a Dirichlet condition (see [9]) and choosing $x=y$, we deduce that $\Theta u(t, x) \leq \Theta u(s, x)+$ $\nu+C_{\nu}(s-t)$. We can argue similarly to obtain the other inequality and prove that $|\Theta u(t, x)-\Theta u(s, x)| \leq \nu+C_{\nu}|s-t|$ for all $t, s, x$. The proof of Proposition 4.1 is complete.

\section{B Proof of the measurability of $\sigma_{\varepsilon}($.}

For $t \in[0, T]$, define $\sigma_{\varepsilon}^{n}(t):=\sup _{d_{\varepsilon}^{n}(t)} \Psi(t, x, y, r, r, p, X, Y)$. Let us prove that $\sigma_{\varepsilon}^{n}($.$) is measurable. For a set A$ of a metric space $E$, define

$$
\mathbb{I}_{A}(x):=\left\{\begin{array}{cc}
0 & \text { if } x \in A, \\
-\infty & \text { if not. }
\end{array}\right.
$$

We let $A$ denote the set of $(X, Y) \in \mathbb{S}^{2 N}$ such that (3.1) holds true. For $t \in[0, T]$, we let $B(t)$ (resp. $C(t))$ denote the set of $r \in \mathbb{R}$ (resp. $(x, y) \in \mathbb{R}^{2 N}$ ) such that $r \leq\left\|u_{1}(t, .)\right\|_{\infty} \vee\left\|u_{1}(t, .)\right\|_{\infty}\left(\operatorname{resp} . \frac{|x-y|^{2}}{4 \varepsilon} \leq m_{2 \varepsilon}(t)-m_{\varepsilon}(t)+\frac{1}{n}\right)$. We see that

$$
\begin{aligned}
\sigma_{\varepsilon}^{n}(t)= & \sup _{(x, y, r, X, Y) \in \mathbb{R}^{2 N} \times \mathbb{R} \times A}\left\{\Psi\left(t, x, y, r, r, \frac{x-y}{\varepsilon}, X, Y\right)\right. \\
& \left.+\mathbb{I}_{B(t)}(r)+\mathbb{I}_{C(t)}(x, y)\right\} .
\end{aligned}
$$

For each $t \in[0, T], \overline{\left(\stackrel{\circ}{\left({ }^{\prime}(t)\right)}\right.}=C(t)$ and either $B(t)=\{0\}$ or $\overline{(\stackrel{\circ}{B(t)})}=B(t)$; this implies that

$$
\sigma_{\varepsilon}^{n}(t)=\sup _{\mathbb{Q}^{2 N} \times \mathbb{Q} \times D}\left\{\Psi\left(t, x, y, r, r, \frac{x-y}{\varepsilon}, X, Y\right)+\mathbb{I}_{B(t)}(r)+\mathbb{I}_{C(t)}(x, y)\right\},
$$


where $D$ is a countable dense subset of $A$. Moreover, $t \rightarrow\left\|u_{1}(t, .)\right\|_{\infty}$ and $m_{i \varepsilon}($.$) are measurable as l.s.c. functions (i=1,2)$. Consequently, for each $(x, y, r, X, Y) \in \mathbb{Q}^{2 N} \times \mathbb{Q} \times D$, the function

$$
t \rightarrow \Psi\left(t, x, y, r, r, \frac{x-y}{\varepsilon}\right)+\mathbb{I}_{B(t)}(r)+\mathbb{I}_{C(t)}(x, y)
$$

is measurable. The function $\sigma_{\varepsilon}($.$) then is measurable as countable supremum of$ measurable functions. Since $\sigma_{\varepsilon}()=.\inf _{\mathbb{N}^{*}} \sigma_{\varepsilon}^{n}($.$) , we have established the measur-$ ability of $\sigma_{\varepsilon}($.$) .$

\section{Acknowledgments}

The author would like to thank J. Droniou and C. Imbert for the fruitful discussions we had together.

\section{References}

[1] O. AlvarEZ, P. CARDAliaguet and R. MONNEAU, Existence and uniqueness for dislocation dynamics with nonnegative velocity, Interfaces Free Bound 7(4) (2005), 415-434.

[2] O. ALVAREZ and A. TOURIN, Viscosity solutions of nonlinear integrodifferential equations, Ann. Inst. H. Poincaré Anal. Non Linéaire, 13(3) (1996), 293-317.

[3] O. ALVAREZ, P. HOCH, Y. LE BOUAR and R. MONNEAU, Dislocation dynamics: short time existence and uniqueness of the solution, Arch. Rational Mech. Ana. 181(3) (2006), 449.

[4] L. ALVAREZ, P.-L. LIONS and J.-M. MOREL, Image selective smoothing and edge detection by nonlinear diffusion. II, SIAM J. Numer. Anal., 29(3) (1992), 845-866.

[5] J.-P. AUBIN, Initiation à l'analyse appliquée, Masson (1994)

[6] G. BARLES, S. BITON and O. LEY, A Geometrical Approach to the Study of Quasilinear Parabolic Equations in $\mathbb{R}^{N}$ Arch. Rational. Mech. Ana., 162(4) (2002), 287-325.

[7] A. BENSOUSSAN and J.-L. LIONS, Contrôle Impulsionnel et Inéquations QuasiVariationnelles, Dunod, Paris (1982).

[8] M. G. CRANDALL and H. ISHII, The maximum principle for semicontinuous functions, Differential Integral Equations 3 (1990), 1001-1014. 
[9] M. G. CRANDALL, H. ISHII and P.-L. LIONS, User's guide to viscosity solutions of second order partial differential equations, Bull. Amer. Math. Soc. (N.S.), 27 (1992), 1-67.

[10] E. R. JAKOBSEN and K. H. KARLSEN, Continuous Dependance Estimates for Viscosity Solutions of Fully Nonlinear Degenerate Parabolic Equations, Journal of Differential Equations 183 (2002), 497-525.

[11] E. R. JAKOBSEN and K. H. KARLSEN, Continuous dependence estimates for viscosity solutions of integro-PDEs, Journal of Differential Equations 212(2) (2005), 278-318.

[12] N. FORCADEL, Dislocation dynamics with a mean curvature term: short time existence and uniqueness, Preprint (2005).

[13] C. IMBERT, A non-local regularization of first order Hamilton-Jacobi equations, Journal of Differential Equations 211 (2005), 214-246.

[14] A. SAYAH, Equations d'Hamilton-Jacobi du premier ordre avec termes intégro-différentials, Parties I et II, Comm P.D.E. , 16 (1991), 1057-1093.

[15] P. E. SOUGANIDIS, Existence of viscosity solutions of Hamilton-Jacobi equations, J. of Differential Equations 56 (1985), 345-390.

[16] N. YAMADA, Viscosity solutions for integro-differential equations of Volterra type, Gatuko International Series Mathematical Sciences and Applications, 7 (1995), 441-446.

Received 5 October 2005; accepted 17 June 2006

Published Online First 21 September 2007

To access this journal online: http://www.birkhauser.ch 